\title{
Treatments for chronic myeloid leukemia: a qualitative systematic review
}

\author{
This article was published in the following Dove Press journal: \\ Journal of Blood Medicine \\ 2 August 2012 \\ Number of times this article has been viewed
}

\author{
Roxanne Ferdinand' \\ Stephen A Mitchell ${ }^{2}$ \\ Sarah Batson ${ }^{2}$ \\ Indra Tumur' \\ 'Pfizer, Tadworth, UK; ${ }^{2}$ Abacus \\ International, Bicester, UK
}

Correspondence: Roxanne Ferdinand Pfizer, Walton Oaks, Dorking Road, Walton-on-the-Hill, Tadworth,

Surrey KT20 7NS, UK

$\mathrm{Tel}+44$ I737 33। 410

Fax +44 I869 323248

Email roxanne.ferdinand@pfizer.com
Background: Chronic myeloid leukemia (CML) is a myeloproliferative disorder of blood stem cells. The tyrosine kinase inhibitor (TKI) imatinib was the first targeted therapy licensed for patients with chronic-phase CML, and its introduction was associated with substantial improvements in response and survival compared with previous therapies. Clinical trial data are now available for the second-generation TKIs (nilotinib, dasatinib, and bosutinib) in the first-, second-, and third-line settings. A qualitative systematic review was conducted to qualitatively compare the clinical effectiveness, safety, and effect on quality of life of TKIs for the management of chronic-, accelerated-, or blast-phase CML patients.

Methods: Included studies were identified through a search of electronic databases in September 2011, relevant conference proceedings and the grey literature.

Results: In the first-line setting, the long-term efficacy (up to 8 years) of imatinib has been confirmed in a single randomized controlled trial (International Randomized Study of Interferon [IRIS]). All second-generation TKIs reported lower rates of transformation, and comparable or superior complete cytogenetic response (CCyR), major molecular response (MMR), and complete molecular response rates compared with imatinib by 2-year follow-up. Each of the second-generation TKIs was associated with a distinct adverse-event profile. Bosutinib was the only second-generation TKI to report quality-of-life data (no significant difference compared with imatinib treatment). Data in the second- and third-line setting confirmed the efficacy of the second-generation TKIs in either imatinib-resistant or -intolerant patients, as measured by CCyR and MMR rates.

Conclusion: Data from first-line randomized controlled trials reporting up to 2-year follow-up indicate superior response rates of the second-generation TKIs compared with imatinib. Current evidence from single-arm studies in the second-line setting confirm that nilotinib, dasatinib, and bosutinib are valuable treatment options for the significant subgroup of patients who are intolerant or resistant to imatinib treatment.

Keywords: chronic myeloid leukemia, imatinib, nilotinib, dasatinib, bosutinib

\section{Introduction/background}

Chronic myeloid leukemia or chronic myelogenous leukemia (CML) is a myeloproliferative disorder of blood stem cells. It is primarily due to a single genetic anomaly: a reciprocal chromosomal translocation between the $C$ - $A B L$ (Abelson leukemia virus) oncogene on chromosome 9 and the $B C R$ (breakpoint cluster region) on chromosome 22. ${ }^{1,2}$ The resulting $B C R-A B L$ gene encodes a fusion tyrosine kinase, which causes cell-cycle deregulation and apoptosis, as well as affecting differentiation and DNA repair. ${ }^{3}$ 
The incidence of CML ranges from 0.6 to 2.0 cases per 100,000 per year. ${ }^{4}$ Since the introduction of tyrosine kinase inhibitors (TKIs), prevalence rates have increased (due to their efficacy in controlling CML). ${ }^{4}$ The median age of onset of CML was reported to be between 45 and 55 years in 2001,5 but has more recently been reported to be 66 years. ${ }^{6}$

Early treatments for CML included chemotherapeutic agents such as hydroxyurea and busulfan, which were able to control the symptoms of the disease but did not slow disease progression. The introduction of interferon- $\alpha$ (IFN- $\alpha$ ) and stem cell transplantation enabled patients to achieve cytogenetic responses and durable remission. ${ }^{7}$ However, increasing understanding of the abnormal activity of the BCR-ABL protein and its role in CML led to the development of targeted therapies such as TKIs, eg, imatinib, dasatinib, nilotinib, and bosutinib. Imatinib was the first targeted therapy licensed for patients with chronic-phase (CP) CML, although dasatinib and nilotinib have also received approval in this setting. Dasatinib and nilotinib are extensively used in the secondline setting for patients with intolerance and/or resistance to imatinib. They have recently also received regulatory approval in the US, the EU, and Japan in the first-line setting. Bosutinib has been shown to be efficacious with an acceptable safety profile in an open-label phase 2 trial in the second and third line, ${ }^{8-10}$ as well as in an ongoing phase 3 trial in patients with newly diagnosed CP CML..$^{11,12}$

\section{Objective}

To provide a qualitative overview of the clinical effectiveness, safety, and quality of life of TKI treatments in CP, accelerated- and blast-phase (AP/BP) CML patients.

\section{Methods}

\section{Study inclusion criteria}

Inclusion criteria are detailed in Table 1. Only randomized controlled trials (RCTs) evaluating a TKI, as well as registrational studies of TKIs, published before September 2011, were included. Participants had to be adults ( $\geq 18$ years) with chronic, AP, and/or BP CML. First to third-line treatment with bosutinib, imatinib, dasatinib or nilotinib was considered. Studies on IFN- $\alpha$ and older agents as well as studies on stem cell transplantation were excluded. There were no restrictions placed on comparators used in the studies.

Efficacy outcomes were included, but were not restricted to duration and time to response, response rates (cytogenetic, molecular, and hematological), overall survival (OS), eventfree survival (EFS), time to treatment failure (TTF), time to and
Table I Summary of inclusion and exclusion criteria for the systematic review

\begin{tabular}{|c|c|}
\hline Study design & $\begin{array}{l}\text { - Randomized controlled trials of parallel or } \\
\text { crossover design } \\
\text { - Registrational single arm studies } \\
\text { Relevant systematic reviews/meta-analyses were } \\
\text { identified. Reference lists were checked to ensure } \\
\text { all relevant studies were included in the review. }\end{array}$ \\
\hline Population & $\begin{array}{l}\text { - Adult patients ( } \geq 18 \text { years) with chronic-, } \\
\text { accelerated-, and/or blast-phase CML } \\
\text { - Treatment-naïv and/or newly diagnosed } \\
\text { Ph-chromosome-positive patients for the first-line } \\
\text { setting } \\
\text { - Pretreated and intolerant/resistant patients } \\
\text { for the } 2 \text { nd-/3rd-line setting }\end{array}$ \\
\hline Interventions & $\begin{array}{l}\text { First-line therapy: } \\
\text { - Imatinib (CP standard dose } 400 \mathrm{mg} \text { OD up to } \\
400 \mathrm{mg} \text { BID; AP/BC } 600 \mathrm{mg} \text { OD up to } 400 \mathrm{mg} \text { BID) } \\
\text { - Dasatinib (CP } 100 \mathrm{mg} / \mathrm{day} \text {; CP } 70 \mathrm{mg} \text { BID) } \\
\text { - Nilotinib ( } 400 \mathrm{mg} \text { BID) } \\
\text { - Bosutinib (standard dose, } 500 \mathrm{mg} / \text { day), } \\
\text { Second-line therapy: } \\
\text { - Imatinib } \\
\text { - Imatinib-intolerant: dasatinib and nilotinib } \\
\text { - Imatinib resistance: dasatinib and nilotinib } \\
\text { - Imatinib resistance: bosutinib } \\
\text { Third-line therapy: } \\
\text { - Dasatinib } \\
\text { - Nilotinib } \\
\text { - Bosutinib }\end{array}$ \\
\hline Outcomes & $\begin{array}{l}\text { Included, but not restricted to: } \\
\text { Efficacy } \\
\text { - Treatment response rates (including molecular, } \\
\text { cytogenetic and hematologic responses) } \\
\text { - Time to and duration of response } \\
\text { - Transformation rate to AP or BP } \\
\text { - Overall survival } \\
\text { - Event-free survival } \\
\text { - Progression-free survival } \\
\text { - Time to treatment failure } \\
\text { - Health-related quality of life } \\
\text { Safety/tolerability } \\
\text { - Adverse events (all grades) } \\
\text { - Incidence of serious adverse events }\end{array}$ \\
\hline $\begin{array}{l}\text { Language of } \\
\text { publication }\end{array}$ & No restriction \\
\hline
\end{tabular}

Abbreviations: $\mathrm{AP}$, accelerated phase; $\mathrm{BP}$, blast phase; $\mathrm{OD}$, once daily; $\mathrm{CP}$, chronic phase; CML, chronic myeloid leukemia; BID, twice daily

rate of transformation to $\mathrm{AP}$ or $\mathrm{BP}$, and health-related quality of life (HRQoL). Reported safety outcomes included adverse events (AE) (all grades) and the incidence of serious AEs.

\section{Search strategy}

The following electronic databases were searched: the Cochrane Library (incorporating the Central Register of Controlled Trials, Central), OVID Medline, and OVID Embase. No restrictions on date of publication or language were applied. 
Search terms included both free text and Medical Subject Headings terms (eg, leukemia, myelogenous, myeloid, chronic, imatinib, dasatinib, nilotinib, bosutinib). The following conference proceedings were also searched (2007-2011): American Society of Hematology, American Society of Clinical Oncology, and the European Hematology Association. Pfizer provided copies of two conference posters, the abstracts of which had been identified in the database searches. ${ }^{11,12}$

\section{Quality assessment}

The methodological quality of RCTs was assessed independently by two reviewers, according to methods recommended in section six of the Cochrane Handbook for Systematic Reviews of Interventions, version 5.1.0. ${ }^{13}$ The likelihood of bias was assessed according to three criteria: adequacy of randomisation and allocation concealment procedures, adequacy of blinding procedures, and completeness of follow-up.

\section{Results}

Electronic and manual searches identified 3248 potentially relevant publications, of which 3186 were excluded on the basis of title and abstract. Upon examination of the full texts, a further 47 were excluded. Thirty-one additional publications were identified via hand searching. In total, 46 publications, describing eleven RCTs and twelve single-arm studies, were included for detailed analysis (Figure 1). Of the 11 RCTs identified, ${ }^{11,14-23}$ eight investigated first-line ${ }^{11,14-20}$ and three second-line treatments. ${ }^{21-23}$ Only CP CML patients were included in the RCTs. No RCTs on third-line treatments were identified for inclusion in this systematic review, although one second-line trial included extensively pretreated patients..$^{21,24}$ Of the singlearm studies, eight investigated second-line treatments, ${ }^{9,25-31}$ three third-line treatments, ${ }^{10,32,33}$ and one study enrolled both second- and third-line patients. ${ }^{34} \mathrm{CP}$ patients were enrolled

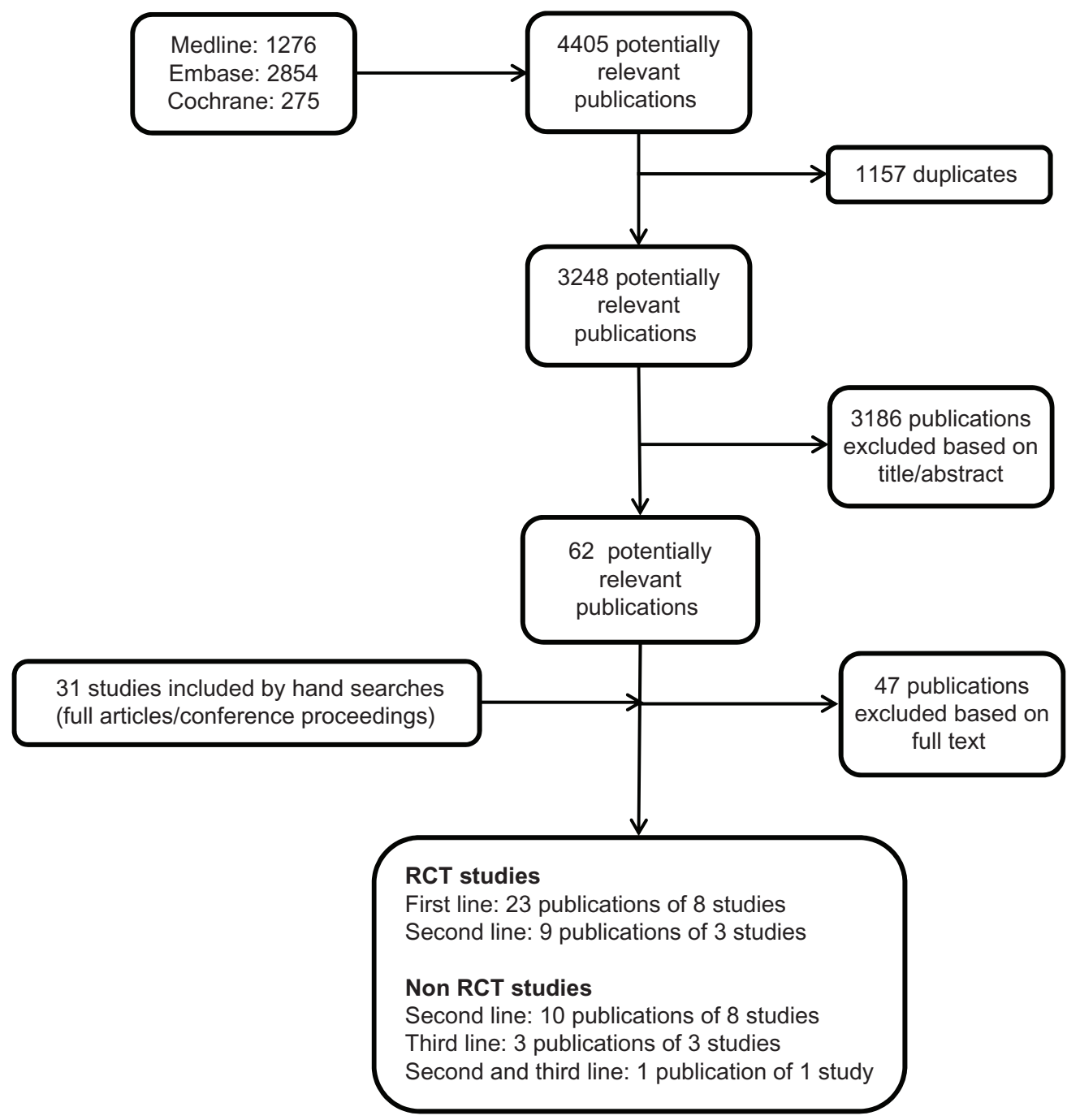

Figure I Trial flow. 
in six trials, ${ }^{9,10,25,30,31,33}$ AP patients in one trial, ${ }^{26} \mathrm{BP}$ patients in one trial, ${ }^{27}$ acute lymphoblastic leukemia (ALL) patients in a subgroup of one trial, ${ }^{29}$ and mixed-patient populations were enrolled in three trials. ${ }^{28,32,34}$

\section{First-line treatments}

One study (International Randomized Study of Interferon and STI571 [IRIS]) (eight publications) compared imatinib with IFN- $\alpha$ plus cytarabine. ${ }^{14,35-41}$ Two trials (Dasatinib vs Imatinib Study In Treatment-Naïve CML [DASISION] ${ }^{15,42,43}$ and $\mathrm{S} 0325^{16}$ ), compared imatinib with dasatinib. Imatinib was also compared with nilotinib in one trial (Evaluating Nilotinib Efficacy and Safety in Clinical Trials - Newly Diagnosed Patients [ENESTnd]). ${ }^{17,44-46}$ A single RCT (Bosutinib Efficacy and Safety in Newly Diagnosed Chronic Myeloid Leukemia [BELA]) compared bosutinib with imatinib. ${ }^{11,12,47}$ Different doses/administration schedules of imatinib were compared in two trials. ${ }^{18,19,48}$ Different dose regimens of dasatinib were compared in one trial. ${ }^{20}$ An overview of the included publications, including patient baseline characteristics and main efficacy outcomes, is provided in Tables 2 and 3.

\section{Imatinib versus interferon- $\alpha$ plus cytarabine (IRIS)}

A total of eight publications reported results from a prospective, multicenter, open-label, phase 3 RCT comparing imatinib $400 \mathrm{mg} /$ day with IFN- $\alpha$ (target dose of $5 \mathrm{MU} / \mathrm{m}^{2}$ / day) plus cytarabine $\left(20 \mathrm{mg} / \mathrm{m}^{2}\right.$ for 10 days per month, once maximum IFN- $\alpha$ dose was reached). Overall, 1106 patients were randomized in the IRIS trial.

In 2003, O'Brien et $\mathrm{a}^{14}$ published the first data (after a median follow-up of 19 months): estimated complete cytogenetic response (CCyR) rates at 18 months were $76.2 \%$ with imatinib versus $14.5 \%$ with IFN- $\alpha$ plus cytarabine $(P<0.001)$. At 18 months, the estimated rate of freedom from progression to AP or BP was $96.7 \%$ with imatinib and $91.5 \%$ with the combination-therapy $(P<0.001)$. The most common AEs reported with imatinib were superficial edema, nausea, muscle cramps, and rashes. Rates of discontinuation (for any reason) and crossover to the alternative treatment were higher in the IFN- $\alpha$ group than in the imatinib group (discontinuations $12.3 \%$ with imatinib vs $31.6 \%$ with IFN- $\alpha$; crossover $2.0 \%$ with imatinib vs $57.5 \%$ with IFN- $\alpha$ ). Further results from this study were published in $2006,{ }^{36}$ when (after a median follow-up of 60 months) cumulative CCyR rates were estimated at $69 \%$ by 12 months and $87 \%$ by 60 months. Newly occurring or worsening grade 3/4 AEs were infrequent after 4 years of therapy, and there had been no change in the AE profile. Hochhaus et $\mathrm{al}^{37}$ published 6-year follow-up data (focusing on patients treated with imatinib), and they reported no further cases of disease progression and an unchanged $\mathrm{AE}$ profile. Seven-year data reported a best CCyR of $82 \%$, and a total of 317 (57\%) of all randomized patients remained on imatinib and were in CCyR (38). At 8 years, ${ }^{39} 55 \%$ of patients initially randomized to imatinib were still on study treatment. The estimated OS rate was $85 \%$ (or $93 \%$ for CML-related deaths only). The authors also concluded that most progression events occurred within 3 years of imatinib treatment, with a very low risk of progression thereafter. A retrospective analysis of the trial data ${ }^{35}$ favored imatinib dose escalation for the initial treatment of CML patients with suboptimal CCyR or cytogenetic resistance.

Guilhot et $\mathrm{al}^{41}$ investigated the relationship between time to CCyR and long-term outcomes in patients treated with imatinib. Results were reported in an abstract and indicated that the durability of major cytogenetic response did not differ significantly, regardless of when CCyR was achieved $(P=0.76)$ in patients who were treated for at least 1 year and achieved CCyR during therapy. Patients who did not achieve CCyR had significantly worse outcomes than those who did achieve CCyR $(P<0.001)$. However, there was a nonstatistically significant difference observed when categorized according to time to response.

In 2010, Hughes et $\mathrm{al}^{40}$ published an analysis of the longterm prognostic significance of an early molecular response (in imatinib-treated patients taking part in the IRIS trial). The authors found that EFS was shorter and rates of progression higher in patients with $B C R-A B L$ transcripts $>10 \%$ at 6 months and $>1 \%$ at 12 months. Also, only $3 \%$ of patients who had achieved a major molecular response (MMR) by 18 months lost CCyR by 7 years, compared with $26 \%$ of patients without MMR (but with CCyR) at 18 months ( $P<0.001)$. Of patients with MMR (at 12 or 18 months), $99 \%$ did not progress to AP or BP, compared with approximately $90 \%$ of patients without MMR (at 12 or 18 months). The authors concluded that molecular response status early during treatment may serve as a predictor of optimal response to therapy.

\section{Nilotinib versus imatinib (ENESTnd)}

A single phase 3, open-label RCT (ENESTnd) compared nilotinib (300 mg or $400 \mathrm{mg}$ twice daily [BID]) with imatinib $400 \mathrm{mg}$ once daily (OD) in 846 patients. ${ }^{17}$ Rates of MMR at 12 months were significantly higher in the nilotinib treatment groups (44\% with $300 \mathrm{mg}$, and 43\% with $400 \mathrm{mg}$ ) compared with imatinib (22\%, $P<0.001$ for both comparisons). The difference in cumulative CCyR rates by 12 months was also 


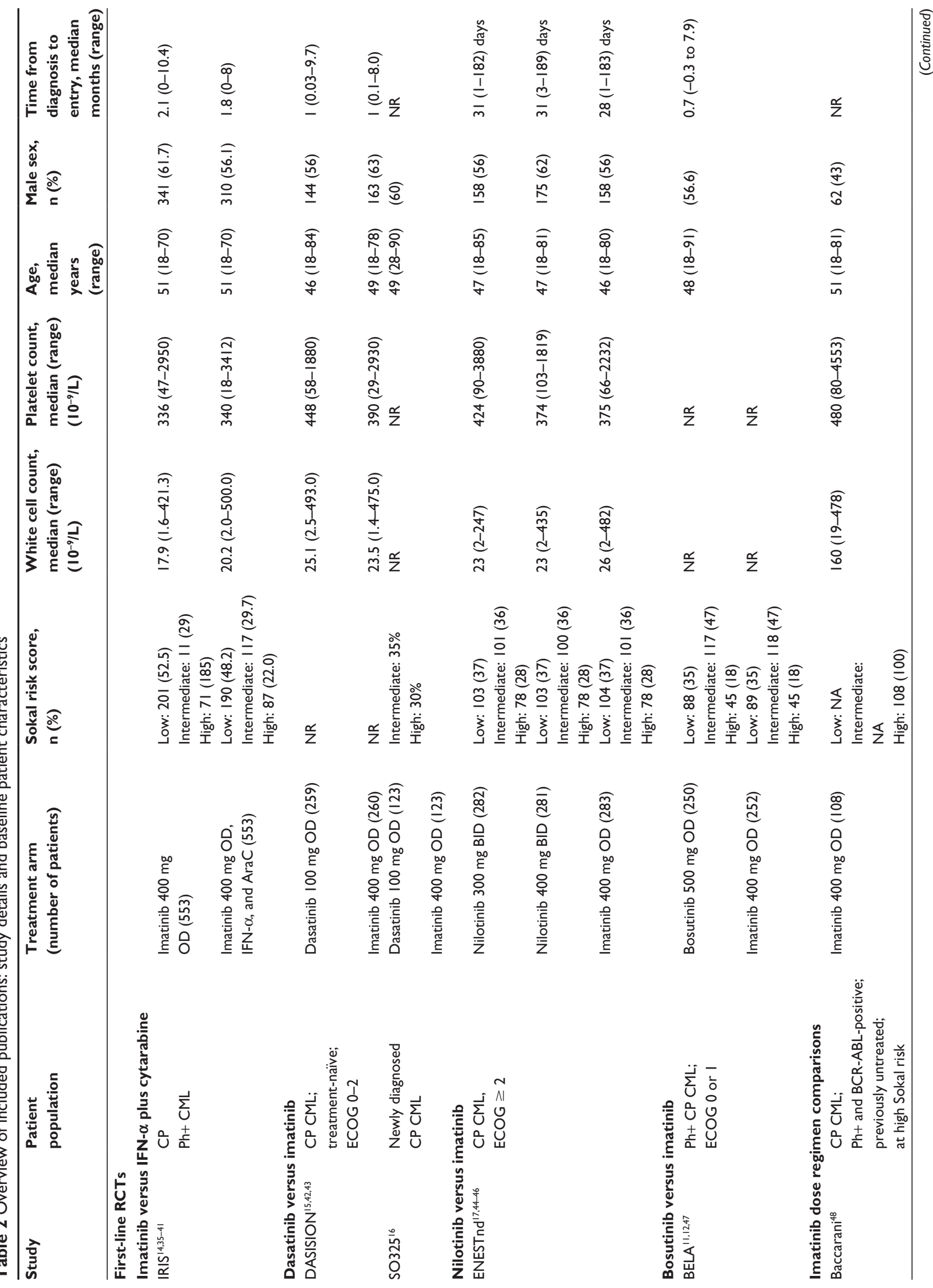




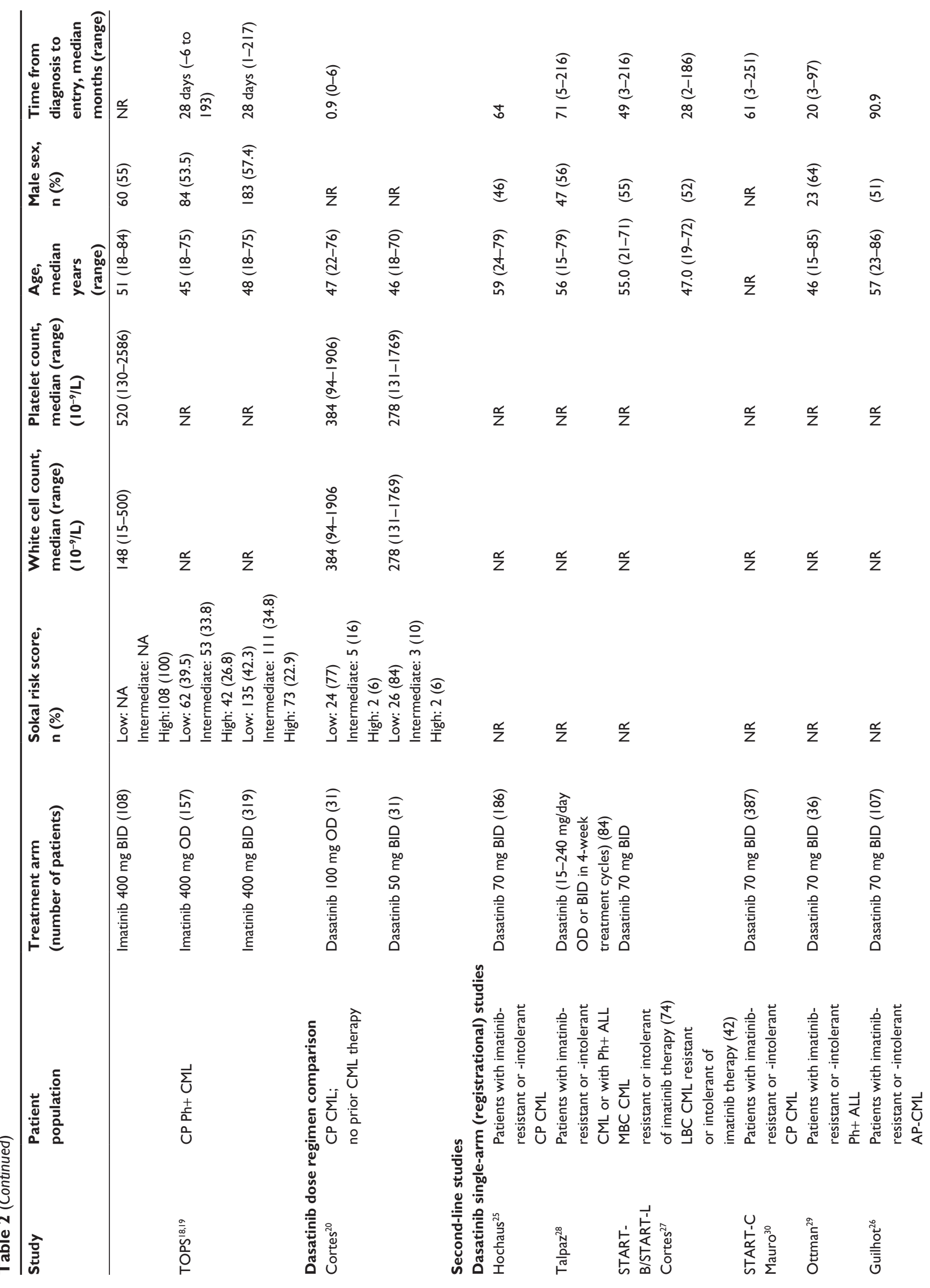




\section{兴}

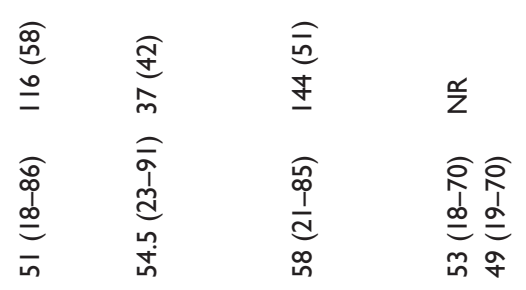

$\stackrel{\propto}{z}$

$\stackrel{o}{z}$

号

产

品 品

Z

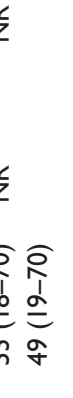

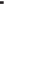




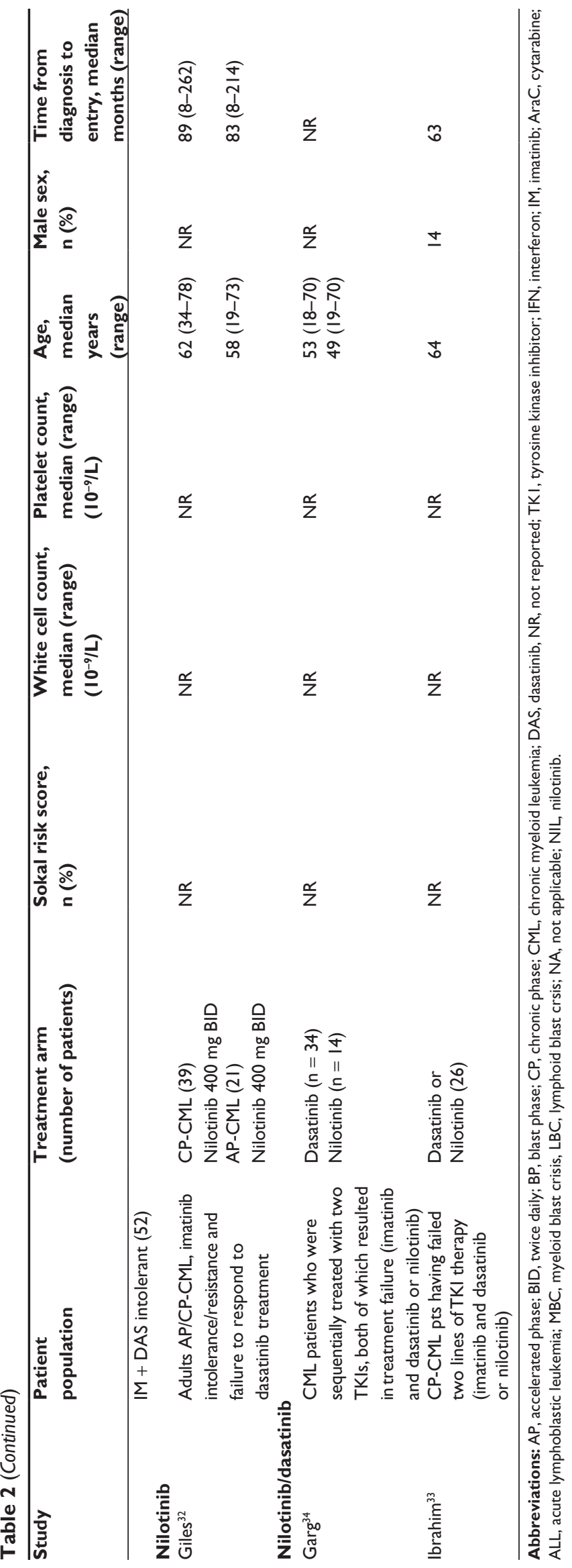

statistically significant: $80 \%$ and $78 \%$ for nilotinib $300 \mathrm{mg}$ and $400 \mathrm{mg}$, respectively, compared with $65 \%$ for imatinib $(P<0.001$ for both comparisons). Transformation to AP or BP occurred in eleven patients (4\%) receiving imatinib, two patients $(<1 \%)$ receiving nilotinib $300 \mathrm{mg}$, and one patient $(<1 \%)$ on nilotinib $400 \mathrm{mg}$. There were some differences in the AE profile between nilotinib and imatinib. With nilotinib, the incidence of rash, headache, and pruritus increased, as did levels of bilirubin, lipase, alanine aminotransferase (ALT) and aspartate aminotransferase (AST). There were, however, fewer cases of nausea, vomiting, diarrhea, muscle spasms, edema, neutropenia, and creatinine increase. Discontinuation rates were comparable between the treatment arms. At 18-month follow-up, ${ }^{44} \mathrm{MMR}$ was reported in $66 \%$ of patients treated with nilotinib $300 \mathrm{mg}$ and $62 \%$ of those receiving nilotinib $400 \mathrm{mg}$, compared with $40 \%$ of imatinib-treated patients $(P<0.0001$ vs imatinib for both nilotinib doses). A complete molecular response (CMR) was reported in $21 \%$ of patients treated with nilotinib $300 \mathrm{mg}$ and $17 \%$ of those receiving nilotinib $400 \mathrm{mg}$, compared with $6 \%$ of imatinibtreated patients $(P<0.0001 \mathrm{vs}$ imatinib for both nilotinib doses). Similarly, CCyR rates of $85 \%(P<0.001)$ and $82 \%$ $(P=0.017)$ were reported for nilotinib $300 \mathrm{mg}$ and $400 \mathrm{mg}$ treated patients, respectively, versus $74 \%$ of imatinib-treated patients. The 24-month follow-up data confirmed the previous results favouring nilotinib. ${ }^{45,46}$ The MMR rates at 24 months were $37 \%$ for imatinib and $62 \%(P<0.001)$ and $59 \%$ $(P<0.001)$ for nilotinib $300 \mathrm{mg}$ and $400 \mathrm{mg}$, respectively. ${ }^{45}$ Rates of CCyR were also significantly better with nilotinib $(87 \%, P=0.0018$ with $300 \mathrm{mg}$, and $85 \%, P=0.016$ with $400 \mathrm{mg}$ ) than with imatinib (77\%). ${ }^{45,46}$ In addition, CMR (4.5-log reduction) at any time was achieved by $26 \%$ and $21 \%$ of nilotinib $300 \mathrm{mg}$ and $400 \mathrm{mg}$-treated patients, respectively, versus $10 \%$ of imatinib-treated patients $(P<0.0001$ vs imatinib for nilotinib $300 \mathrm{mg}$, and $P=0.0004$ for nilotinib $400 \mathrm{mg}) .{ }^{46}$ Progression rates were $4.2 \%$ with imatinib compared with $0.7 \%(P=0.006)$ and $1.1 \%(P=0.020)$ with nilotinib $300 \mathrm{mg}$ and $400 \mathrm{mg}$, respectively. There was, however, no significant difference in OS: $96.3 \%$ for imatinib, and 97.4\% $(P=0.65)$ and $97.8 \%(P=0.21)$ for nilotinib $300 \mathrm{mg}$ and $400 \mathrm{mg}$, respectively. There were no notable changes in the AE profile of nilotinib.

\section{Dasatinib versus imatinib (DASISION, SO325)}

The open-label, phase 3 DASISION RCT (519 patients) compared imatinib $400 \mathrm{mg}$ OD with dasatinib $100 \mathrm{mg}$ OD. ${ }^{15}$ Confirmed cumulative rates of CCyR by 12 months and MMR by 12 months were significantly higher with dasatinib 
Table 3 Overview of included publications - efficacy results

\begin{tabular}{|c|c|c|c|c|}
\hline Study & Publication & CCyR & MMR & Other outcomes reported \\
\hline \multicolumn{5}{|c|}{ First-line RCTs } \\
\hline \multicolumn{5}{|c|}{ Imatinib versus IFN- $\alpha$ plus cytarabine } \\
\hline \multirow[t]{7}{*}{ IRIS } & $\begin{array}{l}\text { O'Brien } \\
\text { et } \mathrm{al}^{14}\end{array}$ & $\begin{array}{l}\text { At I8 months: } \\
\text { - IFN, I4.5\% } \\
\text { - Imatinib, } 76.2 \%, P<0.00 \text { I }\end{array}$ & NR & $\begin{array}{l}\text { PFS rate at } 12 \text { months: } \\
\text { - Imatinib, } 96.6 \% \\
\text { - Imatinib and IFN- } \alpha, 79.9 \%, P<0.00 \text { I } \\
\text { PFS rate at } 18 \text { months: } \\
\text { - Imatinib, } 92.1 \% \\
\text { - Imatinib and IFN- } \alpha, 73.5 \% \text {, } \\
\text { Estimated OS rate at } 18 \text { months: } \\
\text { - Imatinib, } 97.2 \% \\
\text { - Imatinib and IFN- } \alpha, 95.1 \%, P=0.16\end{array}$ \\
\hline & $\begin{array}{l}\text { Druker } \\
\text { et } \mathrm{al}^{36}\end{array}$ & $\begin{array}{l}\text { By } 12 \text { months: } \\
\text { - Imatinib 69\% } \\
\text { By } 60 \text { months: } \\
\text { - Imatinib, 87\% }\end{array}$ & NR & $\begin{array}{l}\text { Estimated PFS at } 60 \text { months: } \\
\text { - Imatinib, } 93 \%(95 \% \mathrm{Cl}, 90-96) \\
\text { Estimated EFS at } 60 \text { months: } \\
\text { - Imatinib } 400 \text { mg OD, } 83 \% \\
\quad(95 \% \mathrm{Cl}, 79-87) \\
\text { Disease progression to AP/BC: } \\
\text { - Imatinib, } 6 \%\end{array}$ \\
\hline & $\begin{array}{l}\text { Hochhaus } \\
\text { et } \mathrm{al}^{37}\end{array}$ & NR & NR & $\begin{array}{l}\text { Estimated PFS at } 7 \text { years: } \\
\text { - Imatinib, } 93 \% \\
\text { Estimated EFS at } 6 \text { years: } \\
\text { - Imatinib, } 67.3 \% \text { (high Sokal risk group) } \\
\text { - Imatinib, } 81.3 \% \text { (intermediate Sokal risk } \\
\text { group) } \\
\text { - Imatinib, } 90.8 \% \text { (low Sokal risk group), } \\
P<0.00 \text { I } \\
\text { OS rate at } 6 \text { years: } \\
\text { - Imatinib, } 76.3 \% \text { (high Sokal risk group) } \\
\text { - Imatinib, } 86.9 \% \text { (intermediate Sokal risk } \\
\text { group) } \\
\text { - Imatinib, } 93.9 \% \text { (low Sokal risk group), } \\
\text { P }<0.00 \text { I } \\
\text { Estimated OS rate at } 8 \text { years: } \\
\text { - Imatinib, } 85 \%\end{array}$ \\
\hline & $\begin{array}{l}\text { O'Brien } \\
\text { et } \mathrm{al}^{38}\end{array}$ & NR & $\begin{array}{l}\text { With imatinib, } \\
\text { at } 12 \text { and } 48 \text { months: } \\
53 \% \text { and } 80 \% \text {, respectively }\end{array}$ & $\begin{array}{l}\text { Estimated EFS at } 7 \text { years: } \\
\text { - Imatinib, } 81 \%\end{array}$ \\
\hline & $\begin{array}{l}\text { Deninger } \\
\text { et } \mathrm{al}^{39}\end{array}$ & NR & NR & $\begin{array}{l}\text { Estimated OS rate at } 8 \text { years: } \\
\text { - Imatinib, } 85 \%\end{array}$ \\
\hline & $\begin{array}{l}\text { Kantarjian } \\
\text { et } \mathrm{al}^{35}\end{array}$ & NR & NR & $\begin{array}{l}\text { Estimated PFS rates at } 12 \text { months after } \\
\text { dose escalations (for } 106 \text { patients with dose } \\
\text { escalations): } \\
\text { - Imatinib, } 94 \% \\
\text { Estimated PFS rates at } 36 \text { months after } \\
\text { dose escalations (for } 106 \text { patients with dose } \\
\text { escalations): } \\
\text { - Imatinib, } 89 \%\end{array}$ \\
\hline & $\begin{array}{l}\text { Guilhot } \\
\text { et } \mathrm{al}^{41}\end{array}$ & NR & NR & $\begin{array}{l}\text { In } 55 \text { I pts of imatinib treatment arm } \\
\text { at } 6 \text { years: } \\
\text { Estimated OS: } 88 \% \\
\text { Estimated EFS: } 83 \% \\
\text { Estimated freedom from progression } \\
\text { to AP/BC: } 93 \% \\
\text { No significant correlation between the } \\
\text { achievement of CCyR and the durability of } \\
\text { MCyR }(P=0.76)\end{array}$ \\
\hline
\end{tabular}


Table 3 (Continued)

\begin{tabular}{|c|c|c|c|c|}
\hline Study & Publication & CCyR & MMR & Other outcomes reported \\
\hline & Hughes et a $\left.\right|^{40}$ & NR & NR & $\begin{array}{l}476 \text { pts of imatinib treatment am: } \\
\text { - EFS was shorter and rates of progression } \\
\text { higher in patients with BCR-ABL } \\
\text { transcripts }>10 \% \text { at } 6 \text { months } \\
\text { and }>1 \% \text { at } 12 \text { months } \\
\text { - } 3 \% \text { of patients who had achieved an } \\
\text { MMR by I } 8 \text { months lost CCyR by } \\
7 \text { years, compared with } 26 \% \text { of patients } \\
\text { without MMR (but with CCyR) at } \\
18 \text { months ( } P<0.00 \mathrm{I}) \\
\text { - patients with MMR (at } 12 \text { or I } 8 \text { months), } \\
99 \% \text { did not progress to AP or BP, } \\
\text { compared with around } 90 \% \text { of patients } \\
\text { without MMR }\end{array}$ \\
\hline \multicolumn{5}{|c|}{ Dasatinib versus imatinib } \\
\hline DASISION & $\begin{array}{l}\text { Kantarjian } \\
\text { et al }{ }^{15}\end{array}$ & $\begin{array}{l}\text { By } 12 \text { months: } \\
\text { - Dasatinib, } 83 \% \\
\text { - Imatinib, } 72 \% \\
\text { By } 12 \text { months: } \\
\text { (confirmed CCyR): } \\
\text { - Dasatinib, } 77 \% \\
\text { - Imatinib, } 66 \%, P=0.007\end{array}$ & $\begin{array}{l}\text { By } 12 \text { months: } \\
\text { - Dasatinib, } 46 \% \\
\text { - Imatinib, } 28 \%, P<0.000 \text { I }\end{array}$ & $\begin{array}{l}\text { Progression to AP/BP by } 12 \text { months: } \\
\text { - Dasatinib, } 2.3 \% \\
\text { - Imatinib, } 3.5 \% \\
\text { Estimated PFS rate at } 12 \text { months: } \\
\text { - Dasatinib, } 96 \% \\
\text { - Imatinib, } 97 \% \\
\text { Estimated OS rate at } 12 \text { months: } \\
\text { - Dasatinib, } 97 \% \\
\text { - Imatinib, } 99 \%\end{array}$ \\
\hline & Shah et $\mathrm{al}^{42}$ & $\begin{array}{l}\text { By } 18 \text { months } \\
\text { (confirmed CCyR): } \\
\text { - Dasatinib, } 78 \% \\
\text { - Imatinib, 70\%, } \\
P=0.04\end{array}$ & $\begin{array}{l}\text { By } 18 \text { months: } \\
\text { - Dasatinib, } 57 \% \\
\text { - Imatinib, } 41 \%, P<0.0002\end{array}$ & $\begin{array}{l}\text { OS rate at } 18 \text { months: } \\
\text { - Dasatinib, } 96 \% \\
\text { - Imatinib, } 97.9 \% \\
\text { Estimated PFS rate at } 18 \text { months: } \\
\text { - Dasatinib, } 94.9 \% \\
\text { - Imatinib, } 93.7 \% \\
\text { Progression to AP/BP by } 18 \text { months: } \\
\text { - Dasatinib, } 1.9 \% \\
\text { - Imatinib, } 3.5 \%\end{array}$ \\
\hline & Kantarjian ${ }^{43}$ & $\begin{array}{l}\text { By } 24 \text { months: } \\
\text { - Dasatinib, 86\% } \\
\text { - Imatinib, 82\% } \\
\text { By } 24 \text { months: } \\
\text { (confirmed CCyR): } \\
\text { - Dasatinib, 80\% } \\
\text { - Imatinib, } 74 \%\end{array}$ & $\begin{array}{l}\text { By } 24 \text { months: } \\
\text { - Dasatinib, } 64 \% \\
\text { - Imatinib, } 46 \% \text {, }\end{array}$ & $\begin{array}{l}\text { Transformation to AP/BP by } 24 \text { months: } \\
\text { - Dasatinib, } 2.3 \% \\
\text { - Imatinib, } 5.0 \% \\
\text { PFS rate at } 24 \text { months: } \\
\text { - Dasatinib, } 93.7 \% \\
\text { - Imatinib, } 92.1 \% \\
\text { FFS rate at } 24 \text { months: } \\
\text { - Dasatinib, } 91.2 \% \\
\text { - Imatinib, } 87.8 \% \\
\text { OS rate at } 24 \text { months: } \\
\text { - Dasatinib, } 95.3 \% \\
\text { - Imatinib, } 95.2 \%\end{array}$ \\
\hline SO325 & Radich $^{16}$ & $\begin{array}{l}\text { At } 12 \text { months (data only } \\
\text { available for } 55 \% \text { of patients): } \\
\text { - Dasatinib, } 57 \% \\
\text { - Imatinib, } 69 \%, P=0.097\end{array}$ & NR & $\begin{array}{l}\text { OS rate at } 12 \text { months: } \\
\text { - Dasatinib, } 100 \% \\
\text { - Imatinib, } 99 \%, P=0.60 \\
\text { PFS rate at } 12 \text { months: } \\
\text { - Dasatinib, } 99 \% \\
\text { - Imatinib, } 96 \%, P=0.19\end{array}$ \\
\hline \multicolumn{5}{|c|}{ Nilotinib versus imatinib } \\
\hline ENESTnd & Saglio et al ${ }^{17}$ & $\begin{array}{l}\text { By } 12 \text { months: } \\
\text { - Nilotinib, } 80 \%(300 \mathrm{mg}) \text {, } \\
78 \% \text { ( } 400 \mathrm{mg}) \\
\text { - Imatinib, 65\%, } \\
P<0.00 \text { I }\end{array}$ & $\begin{array}{l}\text { At I2 months: } \\
\text { - Nilotinib, } 44 \% \text { ( } 300 \mathrm{mg}) \text {, } \\
43 \% \text { ( } 400 \mathrm{mg} \text { ) } \\
\text { - Imatinib, } 22 \%, P<0.000 \text { I }\end{array}$ & $\begin{array}{l}\text { Progression to AP/BP } \\
\text { (median } 14 \text { months treatment): } \\
\text { - Nilotinib } 300 \mathrm{mg} \mathrm{BID,}<1 \% \\
\text { - Nilotinib } 400 \mathrm{mg} \text { BID, }<1 \% \\
\text { - Imatinib, } 4 \%\end{array}$ \\
\hline
\end{tabular}


Table 3 (Continued)

\begin{tabular}{|c|c|c|c|c|}
\hline Study & Publication & CCyR & MMR & Other outcomes reported \\
\hline & $\begin{array}{l}\text { Hughes } \\
\text { et } \mathrm{al}^{44}\end{array}$ & $\begin{array}{l}\text { At I } 8 \text { months overall } \\
\text { best CCyR rates: } \\
\text { - Nilotinib, } 85 \% \\
(300 \mathrm{mg}), P<0.001 \text {, } \\
62 \%(400 \mathrm{mg}), P=0.017 \\
\text { - Imatinib, } 74 \%\end{array}$ & $\begin{array}{l}\text { At I8 months overall best } \\
\text { MMR rates: } \\
\text { - Nilotinib, } 66 \% \text { ( } 300 \mathrm{mg}) \text {, } \\
62 \% \text { ( } 400 \mathrm{mg}) \\
\text { - Imatinib, } 40 \%, P<0.000 \text { I }\end{array}$ & $\begin{array}{l}\text { Progression to AP/BP (median } 18 \text { months } \\
\text { follow-up): } \\
\text { - Nilotinib } 300 \mathrm{mg} \mathrm{BID,} 0.7 \% \\
\text { - Nilotinib } 400 \mathrm{mg} \text { BID, } 0.4 \% \\
\text { - Imatinib, } 4.2 \% \\
\text { Estimated OS at I8 months: } \\
\text { - Nilotinib } 300 \mathrm{mg} \text { BID, } 98.5 \% \text {, } \\
P=0.28 \text { (vs imatinib) } \\
\text { - Nilotinib } 400 \mathrm{mg} \mathrm{BID,} 99.3 \% \\
P=0.03 \text { (vs imatinib) } \\
\text { - Imatinib, } 96.9 \%\end{array}$ \\
\hline & $\begin{array}{l}\text { Kantarjian } \\
\text { et al }{ }^{45,46}\end{array}$ & $\begin{array}{l}\text { By } 24 \text { months: } \\
\text { - Nilotinib } 300 \mathrm{mg}, 87 \% \text {, } \\
P=0.002 \text { vs imatinib } \\
\text { - Nilotinib } 400 \mathrm{mg}, 85 \% \text {, } \\
P=0.02 \text { vs imatinib } \\
\text { - Imatinib, } 77 \%\end{array}$ & $\begin{array}{l}\text { At } 24 \text { months: } \\
\text { - Nilotinib } 300 \mathrm{mg}, 62 \% \text { : } \\
P=0.002 \text { vs imatinib } \\
\text { - Nilotinib } 400 \mathrm{mg}, 59 \% \text { : } \\
\quad P=0.02 \text { vs imatinib } \\
\text { - Imatinib, } 37 \%, P<0.000 \text { I } \\
\text { By } 24 \text { months: } \\
\text { - Nilotinib } 300 \mathrm{mg}, 71 \% \text { : } \\
P=0.002 \text { vs imatinib } \\
\text { - Nilotinib } 400 \mathrm{mg}, 67 \% \text { : } \\
P=0.02 \text { vs imatinib } \\
\text { - Imatinib, } 44 \%, P<0.000 \text { I }\end{array}$ & $\begin{array}{l}\text { Progression to AP/BP at } 24 \text { months: } \\
\text { - Nilotinib } 300 \mathrm{mg} \text { BID, }<1 \% \text {, } \\
P=0.006 \text { vs imatinib } \\
\text { - Nilotinib } 400 \text { mg BID, I. } \% \text {, } \\
P=0.02 \text { vs imatinib } \\
\text { - Imatinib, } 4.2 \% \\
\text { Estimated OS at } 24 \text { months: } \\
\text { - Nilotinib } 300 \text { mg BID, } 97.4 \% \text {, } \\
P=0.65 \text { vs imatinib } \\
\text { - Nilotinib } 400 \text { mg BID, } 97.8 \% \\
P=0.21 \text { vs imatinib } \\
\text { - Imatinib } 400 \text { mg OD, } 96.3 \%\end{array}$ \\
\hline \multicolumn{5}{|c|}{ Bosutinib versus imatinib } \\
\hline \multirow[t]{4}{*}{ BELA } & $\begin{array}{l}\text { Gambacorti- } \\
\text { Passerini } \\
\text { et al" }\end{array}$ & $\begin{array}{l}\text { Only pooled results } \\
\text { reported }\end{array}$ & & \\
\hline & $\begin{array}{l}\text { Gambacorti- } \\
\text { Passerini } \\
\text { et al }{ }^{12}\end{array}$ & $\begin{array}{l}\text { At I year: } \\
\text { - Bosutinib, } 70 \% \\
\text { - Imatinib, } 68 \% \\
\text { Cumulative by I year: } \\
\text { - Bosutinib, } 79 \% \\
\text { - Imatinib, } 75 \%\end{array}$ & $\begin{array}{l}\text { At I year: } \\
\text { - Bosutinib, } 41 \% \\
\text { - Imatinib, } 27 \%, P=0.002 \\
\text { Cumulative by I year: } \\
\text { - Bosutinib, } 47 \% \\
\text { - Imatinib, } 32 \%, P<0.00 \text { I }\end{array}$ & $\begin{array}{l}\text { Transformation to AP/BC (median } \\
\text { treatment duration } 16.6 / 16.8 \text { months): } \\
\text { - Bosutinib, } 2 \% \\
\text { - Imatinib, } 4 \%\end{array}$ \\
\hline & & $\begin{array}{l}\text { At I8 months: } \\
\text { - Bosutinib, } 62 \% \\
\text { - Imatinib, } 67 \% \\
\text { Cumulative by } 18 \text { months: } \\
\text { - Bosutinib, } 79 \% \\
\text { - Imatinib, } 79 \%\end{array}$ & $\begin{array}{l}\text { At I } 8 \text { months: } \\
\text { - Bosutinib, } 46 \% \\
\text { - Imatinib, } 38 \% \\
\text { Cumulative by } 18 \text { months: } \\
\text { - Bosutinib, } 55 \% \\
\text { - Imatinib, } 45 \%, P<0.05\end{array}$ & $\begin{array}{l}\text { Transformation to AP/BC (median } \\
\text { treatment duration 19.3/I9.5 months): } \\
\text { - Bosutinib, } 2 \% \\
\text { - Imatinib, } 5 \% \\
\text { EFS estimates at } 18 \text { months: } \\
\text { - Bosutinib, } 95 \% \\
\text { - Imatinib, } 91 \% \\
\text { OS estimates at } 18 \text { months: } \\
\text { - Bosutinib, } 99 \% \\
\text { - Imatinib, } 95 \%\end{array}$ \\
\hline & $\begin{array}{l}\text { Cortes } \\
\text { et } \mathrm{al}^{47}\end{array}$ & $\begin{array}{l}\text { At } 24 \text { months: } \\
\text { - Bosutinib, } 58 \% \\
\text { - Imatinib, } 65 \% \\
\text { Cumulative by } 24 \text { months: } \\
\text { - Bosutinib, } 79 \% \\
\text { - Imatinib, } 80 \%\end{array}$ & $\begin{array}{l}\text { At } 24 \text { months: } \\
\text { - Bosutinib, } 49 \% \\
\text { - Imatinib, } 42 \% \\
\text { Cumulative by } 24 \text { months: } \\
\text { - Bosutinib, } 61 \% \\
\text { - Imatinib, } 50 \%, P<0.05\end{array}$ & $\begin{array}{l}\text { Transformation to AP/BC ( } 24 \text { months): } \\
\text { - Bosutinib, } 2 \% \\
\text { - Imatinib, } 5 \% \\
\text { OS estimates at } 24 \text { months: } \\
\text { - Bosutinib, } 97 \% \\
\text { - Imatinib, } 95 \%\end{array}$ \\
\hline \multicolumn{5}{|c|}{ Imatinib dose regimen comparisons } \\
\hline & $\begin{array}{l}\text { Baccarani } \\
\text { et al }{ }^{48}\end{array}$ & $\begin{array}{l}\text { At I year: } \\
\text { - Imatinib } 400 \mathrm{mg}, 58 \% \\
\text { - Imatinib } 800 \mathrm{mg}, 64 \% \text {, } \\
P=0.435\end{array}$ & NR & $\begin{array}{l}\text { PFS at } 36 \text { months: } \\
\text { - Imatinib OD, 86\% (95\% Cl, 82-90) } \\
\text { - Imatinib BID, 88\% (95\% Cl, 84-92) } \\
\quad P=0.63 \\
\text { EFS at } 36 \text { months: } \\
\text { - Imatinib OD, } 66 \%(95 \% \mathrm{Cl}, 6 \mathrm{I}-7 \mathrm{I}) \\
\quad P=0.89\end{array}$ \\
\hline
\end{tabular}

(Continued) 
Table 3 (Continued)

\begin{tabular}{|c|c|c|c|c|}
\hline Study & Publication & CCyR & MMR & Other outcomes reported \\
\hline & & & & $\begin{array}{l}\text { - Imatinib BID, } 62 \%(95 \% \mathrm{Cl}, 58-68) \\
\text { FFS at } 36 \text { months: } \\
\text { - Imatinib OD, } 74 \%(95 \% \mathrm{Cl}, 70-78) \\
\quad P=0.89 \\
\text { - Imatinib BID, } 72 \%(95 \% \mathrm{Cl}, 66-78) \\
\text { OS at } 36 \text { months: } \\
\text { - Imatinib OD, } 84 \%(95 \% \mathrm{Cl}, 78-90) \\
P=0.79 \\
\text { - Imatinib BID, } 91 \%(95 \% \mathrm{Cl}, 87-94)\end{array}$ \\
\hline \multirow[t]{2}{*}{ TOPS } & $\begin{array}{l}\text { Cortes } \\
\text { et a }\left.\right|^{18}\end{array}$ & $\begin{array}{l}\text { At } 12 \text { months: } \\
\text { - Imatinib } 400 \mathrm{mg}, 66 \% \\
\text { - Imatinib } 800 \mathrm{mg}, 70 \% \text {, } \\
\quad P=0.347\end{array}$ & $\begin{array}{l}\text { At I } 2 \text { months: } \\
\text { - Imatinib } 400 \mathrm{mg}, 40 \% \\
\text { - Imatinib } 800 \mathrm{mg}, 46 \% \text {, } \\
P=0.2035\end{array}$ & $\begin{array}{l}\text { Estimated PFS rate at I8 months: } \\
\text { - Imatinib OD, } 95 \%(95 \% \mathrm{Cl} \text {, } \\
90.2-99.8) \\
\text { - Imatinib BID, } 97.4 \%(95 \% \mathrm{Cl}, 95.3-99.6) \\
\quad P=0.63 \\
\text { Estimated OS rate at I8 months: } \\
\text { - Imatinib OD, } 98.7 \% \\
\text { - Imatinib BID, } 98.2 \%, P=0.56\end{array}$ \\
\hline & $\begin{array}{l}\text { Baccarani } \\
\text { et } \text { al }^{19}\end{array}$ & $\begin{array}{l}\text { At } 24 \text { months: } \\
\text { - Imatinib } 400 \text { mg, 76\% } \\
\text { - Imatinib } 800 \text { mg, 76\% }\end{array}$ & $\begin{array}{l}\text { At } 24 \text { months: } \\
\text { - Imatinib } 400 \mathrm{mg}, 5 \mathrm{l} \% \\
\text { - Imatinib } 800 \mathrm{mg}, 54 \% \text {, } \\
\quad P=0.2035\end{array}$ & $\begin{array}{l}\text { PFS rate at } 24 \text { months: } \\
\text { - Imatinib OD, } 97 \% \\
\text { - Imatinib BID, } 98 \% \\
\text { EFS rate at } 24 \text { months: } \\
\text { - Imatinib OD, } 95 \% \\
\text { - Imatinib BID, } 95 \% \\
\text { OS rate at } 24 \text { months: } \\
\text { - Imatinib OD, } 97 \% \\
\text { - Imatinib BID, } 98 \%\end{array}$ \\
\hline \multicolumn{5}{|c|}{ Dasatinib dose regimen comparison } \\
\hline & $\begin{array}{l}\text { Cortes } \\
\text { et } \mathrm{al}^{20}\end{array}$ & $\begin{array}{l}\text { I2 months: } \\
\text { - Dasatinib OD, 100\% } \\
\text { - Dasatinib BID, } 95 \%\end{array}$ & $\begin{array}{l}\text { I2 months: } \\
\text { - Dasatinib OD, 7I\% } \\
\text { - Dasatinib BID, 7I\% }\end{array}$ & $\begin{array}{l}\text { Projected EFS rate at } 24 \text { months for all } \\
\text { patients: } \\
\text { - } 88 \% \text { ( } 90 \% \text { when excluding the two } \\
\text { patients who experienced relapse due to } \\
\text { noncompliance) }\end{array}$ \\
\hline \multicolumn{5}{|c|}{ Second-line studies } \\
\hline \multicolumn{5}{|c|}{ Dasatinib single-arm (registrational) studies } \\
\hline & Hochaus et $\mathrm{a}^{25}$ & $\begin{array}{l}\text { At } 6 \text { months: } \\
\text { - Overall, } 33 \% \\
\text { - Imatinib resistant } \\
\text { patients, } 22 \% \\
\text { - Imatinib-intolerant } \\
\text { patients, } 56 \% \\
\text { At } 8 \text { months: } \\
\text { - Overall, } 39 \% \\
\text { - Imatinib-resistant } \\
\text { patients, } 28 \% \\
\text { - Imatinib-intolerant } \\
\text { patients, } 64 \%\end{array}$ & NR & NR \\
\hline & Talpaz et al ${ }^{28}$ & $\begin{array}{l}\text { Follow-up duration unclear: } \\
\text { - CP CML patients, } 35 \% \\
\text { - AP CML patients, I8\% } \\
\text { - MBP CML patients, } 26 \% \\
\text { - LBP CML patients, } 30 \%\end{array}$ & NR & NR \\
\hline $\begin{array}{l}\text { START-B/ } \\
\text { START-L }\end{array}$ & Cortes et $\mathrm{a}^{27}$ & $\begin{array}{l}\text { At } 6 \text { months: } \\
\text { - MBC-CML patients, } 27 \% \\
\text { - LBC-CML patients, } 43 \% \\
\text { At } 8 \text { months: } \\
\text { - MBC-CML patients, } 27 \% \\
\text { - LBC-CML patients, } 43 \%\end{array}$ & NR & NR \\
\hline
\end{tabular}


Table 3 (Continued)

\begin{tabular}{|c|c|c|c|c|}
\hline Study & Publication & CCyR & MMR & Other outcomes reported \\
\hline START-C & $\begin{array}{l}\text { Mauro } \\
\text { et } \mathrm{al}^{30}\end{array}$ & $\begin{array}{l}\text { 2-year follow-up rates: } \\
\text { - Overall } 53 \% \\
\text { - Imatinib-intolerant } \\
\text { patients, } 78 \%\end{array}$ & $\begin{array}{l}\text { 2-year follow-up rates: } \\
\text { - Overall } 47 \% \\
\text { - Imatinib-intolerant } \\
\text { patients, } 78 \%\end{array}$ & $\begin{array}{l}\text { PFS at } 2 \text { years: } \\
\text { - Overall } 80 \% \\
\text { - Imatinib-resistant patients, } 75 \% \\
\text { - Imatinib-intolerant patients, } 94 \% \\
\text { OS } 2 \text { years: } \\
\text { - Overall } 94 \% \\
\text { - Imatinib-resistant patients, } 92 \% \\
\text { - Imatinib intolerant patients, } 100 \%\end{array}$ \\
\hline \multirow[t]{2}{*}{ START-L } & Ottman et $\mathrm{a}^{29}$ & $\begin{array}{l}\text { At } 6 \text { months: } \\
\text { - Overall, 58\% } \\
\text { At } 8 \text { months: } \\
\text { - Overall, } 58 \%\end{array}$ & NR & $\begin{array}{l}\text { NEL at } 6 \text { months: } \\
\text { - Overall, II\% } \\
\text { NEL at } 8 \text { months: } \\
\text { - Overall, } 8 \% \\
\text { Median duration of PFS, } 3.3 \text { months }\end{array}$ \\
\hline & Guilhot et $\mathrm{al}^{26}$ & $\begin{array}{l}\text { At } 6 \text { months: } \\
\text { - Imatinib-resistant } \\
\text { patients, } 23 \% \\
\text { - Imatinib-intolerant } \\
\text { patients, } 0 \% \\
\text { - Overall, } 22 \% \\
\text { At } 8 \text { months: } \\
\text { - Overall, } 24 \% \\
\text { - Imatinib-resistant } \\
\text { patients, } 25 \% \\
\text { - Imatinib-intolerant } \\
\text { patients, } 13 \%\end{array}$ & NR & NR \\
\hline \multicolumn{5}{|c|}{ Bosutinib single-arm (registrational) study } \\
\hline \multirow[t]{2}{*}{ NCT0026I846 } & Cortes et $\mathrm{al}^{51}$ & $\begin{array}{l}\text { At } 24 \text { weeks: } \\
\text { - IM-resistant, 23\% } \\
\text { - IM-intolerant, 23\% } \\
\text { - Overall, 23\% } \\
\text { By } 24 \text { weeks: } \\
\text { - IM-resistant, } 41 \% \\
\text { - IM-intolerant, } 41 \% \\
\text { - Overall, } 41 \%\end{array}$ & NR & $\begin{array}{l}\text { PFS at I year: } \\
\text { - IM-resistant, 89\% } \\
\text { - IM-intolerant, 95\% } \\
\text { - Overall, } 91 \% \\
\text { PFS at } 2 \text { years: } \\
\text { - IM-resistant, 73\% } \\
\text { - IM-intolerant, 95\% } \\
\text { - Overall, 79\% } \\
\text { OS at I year: } \\
\text { - Overall, 97\% } \\
\text { OS at } 2 \text { years: } \\
\text { - IM-resistant, 92\% } \\
\text { - IM-intolerant, 89\% } \\
\text { - Overall, } 97 \%\end{array}$ \\
\hline & $\begin{array}{l}\text { Gambacorti- } \\
\text { Passerini et al }\end{array}$ & $\begin{array}{l}\text { 3I. } 6 \text { months median follow-up: } \\
\text { - IM-resistant, } 43 \% \\
\text { - IM-intolerant, } 43 \% \\
\text { - Overall, } 43 \%\end{array}$ & $\begin{array}{l}\text { 3I. } 6 \text { months median follow-up: } \\
\text { - IM-resistant, } 4 \text { I \% } \\
\text { - IM-intolerant, } 46 \% \\
\text { - Overall, } 43 \%\end{array}$ & $\begin{array}{l}\text { Estimated PFS at I year: } \\
\text { - Overall, } 91 \% \\
\text { Estimated PFS at } 2 \text { years: } \\
\text { - Overall, } 81 \% \\
\text { Estimated OS at I year: } \\
\text { - Overall, } 97 \% \\
\text { Estimated OS at } 2 \text { years: } \\
\text { - Overall, } 91 \%\end{array}$ \\
\hline \multicolumn{5}{|c|}{ Nilotinib single-arm (registrational) study } \\
\hline & $\begin{array}{l}\text { Kantarjian et } \\
\mathrm{al}^{31}\end{array}$ & $\begin{array}{l}\text { At least } 6 \text { months follow-up: } \\
\text { - IM-resistant, 30\% } \\
\text { - IM-intolerant, } 35 \% \\
\text { - Overall, } 31 \%\end{array}$ & NR & Estimated I2-month OS rate: $95 \%$ \\
\hline & $\begin{array}{l}\text { Kantarjian et } \\
\mathrm{al}^{50}\end{array}$ & $\begin{array}{l}\text { At least } 24 \text { months follow-up: } \\
\text { - IM-resistant, } 41 \% \\
\text { - IM-intolerant, } 5 \text { I\% } \\
\text { - Overall: } 44 \%\end{array}$ & $\begin{array}{l}\text { At least } 24 \text { months follow-up: } \\
\text { - With baseline CHR: } 38 \% \text {, } \\
P=0.0036 \\
\text { - Without baseline CHR: } 22 \% \\
\text { - Overall: } 28 \%\end{array}$ & $\begin{array}{l}\text { Estimated PFS at } 24 \text { months: } \\
\text { - With baseline CHR: } 77 \% \\
\text { - Without baseline CHR: } 56 \% \\
\text { - Overall: } 64 \% \\
\text { Estimated OS at } 24 \text { months: } \\
\text { - Overall: } 87 \%\end{array}$ \\
\hline
\end{tabular}


Table 3 (Continued)

\begin{tabular}{|c|c|c|c|c|}
\hline Study & Publication & CCyR & MMR & Other outcomes reported \\
\hline \multicolumn{5}{|c|}{ Nilotinib/dasatinib } \\
\hline & Garg et a $\left.\right|^{34}$ & $\begin{array}{l}\text { Best overall CCyR: } \\
\text { - Nilotinib therapy, } 9 \% \\
\text { - Dasatinib, } 14 \%\end{array}$ & $\begin{array}{l}\text { Best overall MMR: } \\
\text { - Nilotinib therapy, I5\% }\end{array}$ & NR \\
\hline \multicolumn{5}{|c|}{ Dasatinib dose/administration schedule comparisons } \\
\hline $\begin{array}{l}\text { CAI80-035/ } \\
\text { NCT00I } 23487\end{array}$ & $\begin{array}{l}\text { Kantarjian } \\
\text { et } \mathrm{al}^{22}\end{array}$ & $\begin{array}{l}\text { - Dasatinib } 140 \text { mg OD, 32\% } \\
\text { - Dasatinib } 70 \text { mg BID, 33\% }\end{array}$ & NR & $\begin{array}{l}\text { Estimated PFS rate at } 12 \text { months: } \\
\text { - Dasatinib OD, } 68 \% \\
\text { - Dasatinib BID, } 69 \% \\
\text { Estimated PFS rate at } 24 \text { months: } \\
\text { - Dasatinib OD, } 51 \% \\
\text { - Dasatinib BID, } 55 \%, P=0.566 \\
\text { Estimated OS rate at } 12 \text { months: } \\
\text { - Dasatinib OD, } 78 \% \\
\text { - Dasatinib BID, } 84 \% \\
\text { Estimated OS rate at } 24 \text { months: } \\
\text { - Dasatinib OD, } 63 \% \\
\text { - Dasatinib BID, } 72 \%, P=0.140\end{array}$ \\
\hline & $\begin{array}{l}\text { Saglio } \\
\text { et } \mathrm{al}^{52}\end{array}$ & $\begin{array}{l}\text { Myeloid blast phase: } \\
\text { - Dasatinib } 140 \text { mg OD, I4\% } \\
\text { - Dasatinib } 70 \text { mg BID, } 2 \text { I\% } \\
\text { Lymphoid blast phase: } \\
\text { - Dasatinib I40 mg OD, 38\% } \\
\text { - Dasatinib } 70 \text { mg BID, 36\% }\end{array}$ & NR & $\begin{array}{l}\text { Estimated PFS rate at } 12 \text { months: } \\
\text { Myeloid blast phase } \\
\text { - Dasatinib OD, } 18 \% \\
\text { - Dasatinib BID, } 25 \% \\
\text { Lymphoid blast phase } \\
\text { - Dasatinib OD, not reached } \\
\text { - Dasatinib BID, } 9 \% \\
\text { Estimated PFS rate at } 24 \text { months: } \\
\text { Myeloid blast phase } \\
\text { - Dasatinib OD, } 11 \% \\
\text { - Dasatinib BID, I8\% } \\
\text { Lymphoid blast phase } \\
\text { - Dasatinib OD, not reached } \\
\text { - Dasatinib BID, not reached } \\
\text { Estimated OS rate at } 12 \text { months: } \\
\text { Myeloid blast phase } \\
\text { - Dasatinib OD, } 34 \% \\
\text { - Dasatinib BID, } 39 \% \\
\text { Lymphoid blast phase } \\
\text { - Dasatinib OD, } 46 \% \\
\text { - Dasatinib BID, } 39 \% \\
\text { Estimated OS rate at } 24 \text { months: } \\
\text { Myeloid blast phase } \\
\text { - Dasatinib OD, } 24 \% \\
\text { - Dasatinib BID, } 28 \% \\
\text { Lymphoid blast phase } \\
\text { - Dasatinib OD, } 21 \% \\
\text { - Dasatinib BID, } 16 \% \\
\end{array}$ \\
\hline NCT00I 23474 & $\begin{array}{l}\text { Shah } \\
\text { et } \mathrm{al}^{23}\end{array}$ & $\begin{array}{l}8 \text { months median: } \\
\text { - Dasatinib } 100 \text { mg OD, } 41 \% \\
\text { - Dasatinib } 50 \text { mg BID, } 42 \% \\
\text { - Dasatinib } 140 \text { mg OD, } 44 \% \\
\text { - Dasatinib } 70 \text { mg BID, } 45 \%\end{array}$ & NR & NR \\
\hline & $\begin{array}{l}\text { Shah } \\
\text { et a }\left.\right|^{54} \\
\text { Shah } \\
\text { et a }\left.\right|^{53}\end{array}$ & $\begin{array}{l}2 \text { years minimum: } \\
\text { - Dasatinib } 100 \text { mg OD, 50\% } \\
\text { - Dasatinib } 50 \text { mg BID, } 54 \% \\
\text { - Dasatinib } 140 \text { mg OD, } 50 \% \\
\text { - Dasatinib } 70 \text { mg BID, 50\% }\end{array}$ & $\begin{array}{l}2 \text { years minimum: } \\
\text { - Dasatinib } 100 \mathrm{mg} \text { OD, } 37 \% \\
\text { - Dasatinib } 50 \mathrm{mg} \text { BID, 38\% } \\
\text { - Dasatinib } 140 \mathrm{mg} \text { OD, } 38 \% \\
\text { - Dasatinib } 70 \mathrm{mg} \text { BID, } 38 \%\end{array}$ & $\begin{array}{l}\text { PFS at } 24 \text { months: } \\
\text { - Dasatinib } 100 \text { mg OD, } 80 \% \\
\text { - All other arms } 75 \%-76 \% \\
\text { Estimated PFS at } 24 \text { months: } \\
\text { - Dasatinib } 100 \text { mg OD, } 80 \% \\
\text { - Dasatinib } 50 \text { mg BID, } 76 \% \\
\text { - Dasatinib I } 40 \text { mg OD, } 75 \% \\
\text { - Dasatinib } 70 \text { mg BID, } 76 \%\end{array}$ \\
\hline
\end{tabular}


Table 3 (Continued)

\begin{tabular}{|c|c|c|c|c|}
\hline Study & Publication & CCyR & MMR & Other outcomes reported \\
\hline & $\begin{array}{l}\text { Shah } \\
\text { et } \mathrm{al}^{55}\end{array}$ & NR & NR & $\begin{array}{l}\text { PFS at } 36 \text { months: } \\
\text { - Dasatinib } 100 \text { mg OD, } 73 \% \\
\text { - Dasatinib } 50 \text { mg BID, } 72 \% \\
\text { - Dasatinib I } 40 \text { mg OD, } 60 \% \\
\text { - Dasatinib } 70 \text { mg BID, } 67 \% \\
\text { OS at } 36 \text { months: } \\
\text { - Dasatinib } 100 \text { mg OD, } 87 \% \\
\text { - Dasatinib } 50 \text { mg BID, } 84 \% \\
\text { - Dasatinib } 140 \text { mg OD, } 80 \% \\
\text { - Dasatinib } 70 \text { mg BID, } 80\end{array}$ \\
\hline & $\begin{array}{l}\text { Shah } \\
\text { et } \mathrm{al}^{56}\end{array}$ & $\begin{array}{l}\text { Best overall response rate } \\
\text { within } 5 \text { years: } \\
\text { - Dasatinib } 100 \mathrm{mg} \text { OD, } 50 \%\end{array}$ & $\begin{array}{l}\text { Within } 5 \text { years: } \\
\text { - Dasatinib } 100 \text { mg OD, } 44 \%\end{array}$ & $\begin{array}{l}\text { PFS at } 60 \text { months: } \\
\text { - Dasatinib } 100 \text { mg OD, } 57 \% \\
\text { OS at } 60 \text { months: } \\
\text { - Dasatinib } 100 \text { mg OD, } 78 \% \\
\text { Transformation to AP } \\
\text { - Dasatinib } 100 \text { mg OD, } 5 \%\end{array}$ \\
\hline \multicolumn{5}{|c|}{ High-dose imatinib versus dasatinib } \\
\hline START-R & $\begin{array}{l}\text { Kantarjian } \\
\text { et } \mathrm{al}^{21}\end{array}$ & $\begin{array}{l}\text { At } 12 \text { weeks: } \\
\text { - Dasatinib, } 22 \% \\
\text { - Imatinib } 800 \mathrm{mg}, 8 \% \text {, } \\
P=0.04 \text { I } \\
\text { I5 months median follow-up: } \\
\text { - Dasatinib, } 40 \% \\
\text { - Imatinib, } 800 \mathrm{mg}, 16 \% \text {, } \\
P=0.004\end{array}$ & $\begin{array}{l}\text { I5 months median follow-up: } \\
\text { - Dasatinib, } 16 \% \\
\text { - Imatinib, } 800 \mathrm{mg}, 4 \% \\
P=0.038\end{array}$ & $\begin{array}{l}\text { PFS (median follow-up I5 months) } \\
\text { - Risk reduction of } 86 \% \text { relative to } \\
\text { high-dose imatinib (HR, } 0.14 ; 95 \% \mathrm{Cl}, 0.1 \\
\text { to } 0.26 ; P<0.00 \mathrm{I}) \\
\text { Treatment failure at } 6 \text { months: } \\
\text { - Dasatinib, I5/I0I, I5\% } \\
\text { - Imatinib, } 37 / 49,76 \% \\
\text { Treatment failure (median, I5 months) } \\
\text { - Dasatinib, } 28 \% \\
\text { - Imatinib, } 82\end{array}$ \\
\hline & $\begin{array}{l}\text { Kantarjian } \\
\text { et } \mathrm{a}^{24}\end{array}$ & $\begin{array}{l}\text { At } 24 \text { months: } \\
\text { - Dasatinib, } 44 \% \\
\text { - Imatinib, } 18 \%, P=0.0025\end{array}$ & $\begin{array}{l}\text { At } 24 \text { months: } \\
\text { - Dasatinib, } 29 \% \\
\text { - Imatinib, } 12 \%, P=0.028\end{array}$ & $\begin{array}{l}\text { PFS at } 24 \text { months: } \\
\text { - Dasatinib OD, } 86 \% \\
\text { - Imatinib OD, } 65 \%, P=0.01\end{array}$ \\
\hline \multicolumn{5}{|c|}{ Third-line single arm studies } \\
\hline & $\begin{array}{l}\text { Khoury } \\
\text { et a }\left.\right|^{57}\end{array}$ & $\begin{array}{l}\text { Median } 28.5 \text {-month follow-up } \\
\text { (best cumulative responses): } \\
\text { - IM+DAS resistant, I4\% } \\
\text { - IM+DAS intolerant, } 28 \% \\
\text { - IM+NIL resistant, } 27 \% \\
\text { - IM+NIL } \pm \text { DAS, } 50 \% \\
\text { - Overall, } 24 \%\end{array}$ & NR & $\begin{array}{l}\text { Transformation to the AP phase, } n=4 \\
\text { Estimated PFS at I year: } \\
\text { - Overall, } 77 \% \\
\text { Estimated PFS at } 2 \text { years: } \\
\text { - Overall, } 73 \% \\
\text { Estimated OS at I year: } \\
\text { - Overall, } 91 \% \\
\text { Estimated OS at } 2 \text { years: } \\
\text { - Overall, } 83 \%\end{array}$ \\
\hline Nilotinib & $\begin{array}{l}\text { Giles } \\
\text { et } \mathrm{al}^{32}\end{array}$ & $\begin{array}{l}\text { Median I2-month follow-up: } \\
\text { - CP CML pts, } 24 \% \\
\text { - AP CML pts, } 0 \%\end{array}$ & & $\begin{array}{l}\text { Discontinuations due to disease } \\
\text { progression: } \\
\text { - II CP, } 8 \text { AP } \\
\text { Estimated OS at } 18 \text { months: } \\
\text { - CP CML pts, } 86 \% \\
\text { - AP CML pts, } 80 \% \\
\text { Estimated PFS at } 18 \text { months: } \\
\text { - } 59 \% \\
\text { Median TTF: } \\
\text { - } 19.5 \text { (range, } 0.9-28.8 \text { ) months }\end{array}$ \\
\hline \multicolumn{5}{|c|}{ Nilotinib/dasatinib } \\
\hline & $\begin{array}{l}\text { Garg } \\
\text { et } \mathrm{al}^{34}\end{array}$ & $\begin{array}{l}\text { Median follow up of } 13 \text { months } \\
\text { (cumulative): } \\
\text { Dasatinib therapy } \\
\text { - CP CML pts, } 31 \% \\
\text { - AP CML pts, } 25 \%\end{array}$ & $\begin{array}{l}\text { Median follow up of I } 3 \text { months } \\
\text { (cumulative): } \\
\text { Dasatinib therapy } \\
\text { - CP CML pts, I } 3 \% \\
\text { - AP CML pts, I3\% }\end{array}$ & $\begin{array}{l}\text { Discontinuations due to transformation: } \\
\text { - Dasatinib, } 21 \% \\
\text { - Nilotinib, } 14 \%\end{array}$ \\
\hline
\end{tabular}


Table 3 (Continued)

\begin{tabular}{|c|c|c|c|c|}
\hline Study & Publication & CCyR & MMR & Other outcomes reported \\
\hline & & - BP CML pts, $20 \%$ & - BP CML pts, I0\% & Median overall survival, 20 months \\
\hline & & Nilotinib therapy & Nilotinib therapy & Median EFS, I 3 months \\
\hline & & - CP CML pts, II\% & - CP CML pts, $33 \%$ & Median PFS, 5 months \\
\hline & & - AP CML pts, $0 \%$ & - AP CML pts, $0 \%$ & \\
\hline & & - BP CML pts, 33\% & - BP CML pts, $0 \%$ & \\
\hline & & Overall & Overall & \\
\hline & & - CP CML pts, $24 \%$ & - CP CML pts, $20 \%$ & \\
\hline & & - AP CML pts, $10 \%$ & - AP CML pts, I0\% & \\
\hline & & - BP CML pts, $23 \%$ & - BP CML pts, $8 \%$ & \\
\hline & Ibrahim & Median 21.5 month follow up: & Median 21.5 month & 30 month probability of EFS, $45.7 \%$ \\
\hline & et $\mathrm{al}^{33}$ & $\begin{array}{l}\text { - } 34.6 \% \\
30 \text { month cumulative incidences: } \\
\text { - } 32.4 \%\end{array}$ & $\begin{array}{l}\text { follow up: } \\
\text { - } 19.2 \% \\
30 \text { month cumulative incidences: } \\
\text { - } 21.1 \%\end{array}$ & 30 month probability of OS, $46.7 \%$ \\
\hline
\end{tabular}

\section{Note: ${ }^{\text {aC }}$ onfirmed complete cytogenetic response.}

Abbreviations: AP, accelerated phase; BC, blast crisis; BP, blast phase; CCyR, complete cytogenetic response; EFS, event free survival; FFP, freedom from progression; FFS, failure-free survival; GM-CSF, granulocyte-macrophage colony stimulating factor; MMR, major molecular response; NEL, no evidence of leukemia; OS, overall survival; PFS, progression-free survival CP, chronic phase; CML, chronic myeloid leukemia; pts, patients; HR, Hazard ratio; NR, not reported; Cl, Confidence interval; CHR, complete hematologic response.

compared with imatinib $(77 \% \mathrm{v} 66 \%, P=0.007$, and $46 \%$ vs $28 \%, P<0.0001)$. This difference in CCyR and MMR was observed across all Hasford risk (HR) categories. In addition, the times to CCyR and MMR were significantly shorter with dasatinib compared with imatinib (HR 1.5, $P<0.0001$, and HR 2.0, $P<0.0001$, respectively). In the dasatinib and imatinib treatment arms, $1.9 \%$ and $3.5 \%$ of patients, respectively, progressed to AP/BP. Rates of progressionfree survival (PFS) at 12 months were similar between the treatment arms. Notable differences in the incidence of AEs were grade $3 / 4$ thrombocytopenia (19\% with dasatinib vs $10 \%$ with imatinib), fluid retention ( $19 \%$ vs $42 \%$ ), nausea ( $8 \%$ vs $20 \%$ ), myalgia ( $6 \%$ vs $12 \%$ ), and muscle inflammation (4\% vs 17\%). Discontinuation rates were similar between the treatment groups. Efficacy and safety results at 18-month follow-up ${ }^{42}$ were consistent with those published at 12 months. The rates of cumulative confirmed CCyR and MMR rates at any time for dasatinib vs imatinib were $78 \%$ vs $70 \%(P=0.0366)$, and $57 \%$ vs $41 \%(P=0.0002)$, respectively. Transformation to AP or BP occurred in six $(2.3 \%)$ patients on dasatinib and nine $(3.5 \%)$ patients on imatinib. After 24 months of minimum follow-up, cumulative rates of confirmed CCyR and MMR were $80 \%$ and $64 \%$ for dasatinib and $74 \%$ and $46 \%$ for imatinib, respectively. ${ }^{43}$ The cumulative rate of CMR (4.5-log reduction) by 24 months was $17 \%$ for dasatinib compared with $8 \%$ for imatinib $(P=0.002)$. The transformation rates for dasatinib and imatinib were $2.3 \%(n=6)$ and $5 \%(n=13)$, respectively (during treatment). With regards to the AE profile, most cytopenias occurred in the first 12 months.
Radich et al ${ }^{16}$ compared imatinib $400 \mathrm{mg}$ OD with dasatinib $100 \mathrm{mg}$ OD in an open-label phase 2 trial, randomising 253 patients. The rates of CCyR were not significantly different between the treatment arms at 12 months (69\% with imatinib, $82 \%$ with dasatinib, $P=0.097$ ), although data were only available for $51 \%$ of patients. Progression data were not reported. In the dasatinib and imatinib arms, 15\% and $11 \%$ of patients, respectively, discontinued due to toxicity. Hematologic AEs were the most common grade 3/4 AEs (eg, thrombocytopenia reported in $18 \%$ and $8 \%$ of patients in the dasatinib and imatinib treatment groups, respectively, $P=0.024$ ). Several nonhematologic grade 4 AEs (not defined in the publication) were reported for $6 \%$ of dasatinib-treated patients and no imatinib patients. Pleural effusion (any grade) was more common with dasatinib compared with imatinib (11\% vs $2 \%, P=0.0017)$.

\section{Bosutinib versus imatinib (BELA)}

A single ongoing, open-label, phase 3 RCT (BELA) (502 patients randomized) of bosutinib (500 mg OD) compared with imatinib (400 mg OD) in the first-line setting has been reported with patients followed for up to 24 months. ${ }^{11,12,47}$ Numerically higher CCyR at 1 year (70\% versus $68 \%)$ and cumulative CCyR rates by 1 year (79\% versus $75 \%)$ were reported for bosutinib-treated versus imatinib-treated patients, although these differences were not statistically significant. ${ }^{12}$ Bosutinib-treated patients reported both significantly higher MMR at 1 year $(41 \%$ vs $27 \%, P=0.002)$ and a 1 -year cumulative MMR rate $(47 \%$ vs $32 \%, P<0.001)$ compared with imatinib-treated patients. ${ }^{12}$ Adverse events that 
were more frequent with bosutinib compared with imatinib at 12 months were mainly gastrointestinal (GI), and included diarrhea (69\% vs $22 \%$ ) and vomiting (32\% vs $14 \%) .{ }^{12}$ With imatinib, the incidence of edema (peripheral, $11 \%$ vs $4 \%$; periorbital, $14 \%$ vs $1 \%$ ), muscle cramps (20\% vs $4 \%$ ), and bone pain ( $10 \%$ vs $4 \%$ ) were higher compared with bosutinib. ${ }^{12}$ Imatinib was also associated with a higher incidence of hematological AEs, including neutropenia (21\% vs 3\%). With regard to laboratory abnormalities, hypophosphatemia was reported more frequently with imatinib compared with bosutinib (17\% vs 4\%), while more bosutinib-treated patients experienced elevated ALT (23\% vs $3 \%)$ or AST (11\% vs $3 \%$ ) compared with imatinib. The rates of discontinuation due to AEs were $22 \%$ for bosutinib and $5 \%$ for imatinib at 18 months (12-month discontinuation data: 19\% vs 6\%); none of these discontinuations were due to diarrhea. ${ }^{12}$ By 18-month follow-up, ${ }^{12}$ the rates of cumulative CCyR were identical for bosutinib and imatinib (both 79\%). However, cumulative CMR (18\% vs 10\%) and MMR (55 vs 45\%) remained significantly in favor of bosutinib. By 24 months, ${ }^{47}$ the reported CCyR was similar for bosutinib (79\%) and imatinib (80\%), although the cumulative MMR remained significantly in favor of bosutinib $(61 \%$ vs $50 \%, P<0.05)$. At 24 months, the times to CCyR and MMR were also significantly in favor of bosutinib $(P<0.001)$. The cumulative rate of CMR (4.5-log reduction) by 24 months was $23 \%$ for bosutinib compared with $16 \%$ for imatinib $(P=0.002)$. Transformation to AP or BP occurred in a numerically higher percentage of patients treated with imatinib at both 12 (4\% vs $2 \%$ with bosutinib, $P=0.053$ ) and 18 and 24 months ( $5 \%$ vs $2 \%$ at both time points). Treatment failure was less common with bosutinib compared with imatinib (4\% vs $13 \%$ ). At the time of reporting, median OS had not been reached at 24-month follow-up (survival estimates for bosutinib and imatinib were $97 \%$ and $95 \%$, respectively). ${ }^{47}$ Patient-reported outcome measures of functioning and health status showed that the different AEs associated with bosutinib and imatinib had minimal overall impact. ${ }^{49}$

Dose-finding studies (imatinib, $\mathrm{n}=2$; dasatinib, $\mathrm{n}=\mathrm{I}$ ) The two imatinib studies (Baccarani et $\mathrm{al}^{48}$ and the Tyrosine Kinase Inhibitor Optimization and Selectivity [TOPS] study $\left.{ }^{18}\right)$ randomized patients $\left(n=200^{48}\right.$ and $\left.n=460^{18}\right)$ to treatment with imatinib $400 \mathrm{mg} /$ day or $800 \mathrm{mg} /$ day. A numerically higher, nonsignificant response was reported for patients in the $800 \mathrm{mg}$ /day group compared with $400 \mathrm{mg} /$ day for both $\mathrm{CCyR}^{18,48}$ and $\mathrm{MMR}^{18}$ at 1 year, which was confirmed at 2-year follow-up in the TOPS study. ${ }^{19}$ With imatinib $800 \mathrm{mg}$ /day, a higher incidence of edema, GI AEs, and rash, as well as grade 3/4 hematological toxicities, was reported with imatinib $800 \mathrm{mg} /$ day.

Cortes et $\mathrm{al}^{20}$ randomized 62 patients to dasatinib $100 \mathrm{mg}$ OD or $50 \mathrm{mg}$ BID. No significant difference between treatment arms was reported with regard to response (CCyR and MMR rates) or the incidence of AEs at 1-year follow-up.

\section{Second-line treatments}

Of the nine included single-arm studies, six were on dasatinib, ${ }^{25-30}$ one reported on nilotinib, ${ }^{31,50}$ one on bosutinib,, 51 and a further study enrolled second-line patients to treatment with either nilotinib or dasatinib. ${ }^{34}$ Three RCTs investigated second-line treatments, all with dasatinib. One trial (START-R) compared high-dose imatinib with dasatinib. ${ }^{21,24}$ Different dose regimens of dasatinib were compared in two trials..$^{22,23,52-56}$ An overview of the included publications, including patient baseline characteristics and main efficacy outcomes, is provided in Tables 2 and 3.

\section{Dasatinib single-arm (registrational) studies}

In an international, open-label, phase 2 study, ${ }^{25} 387$ imatinibresistant or -intolerant CP CML patients were treated with dasatinib. Results were only available for the first 186 patients. The best confirmed CCyR rate at 8-month follow-up was 39\% $(\mathrm{n}=73)$. Rates of MMR and OS were not reported, whereas the PFS rate was $92.4 \%$. Baseline $B C R-A B L$ mutational status was analysed in 180 of 186 patients. With the exception of a single mutation (T315I, identified in 2\% of patients, none of whom attained a major CyR [MCyR] or a complete hematological response), there was no notable influence on the response rate. Imatinib-resistance mutations were only identified in $41 \%$ of patients analyzed. After 8 months, 9\% $(n=6)$ of patients had discontinued due to AEs. The most frequent all-grade AEs were AST and ALT elevation ( $60 \%$ and $52 \%$, respectively), followed by headache (34\%), diarrhoea (30\%), fatigue (28\%), and dyspnea (27\%). Cytopenias were the most common grade 3/4 AEs (ranging from $22 \%$ for anemia to $49 \%$ for neutropenia).

Talpaz et $\mathrm{al}^{28}$ enrolled $40 \mathrm{CP} \mathrm{CML}$ patients, as well as 44 patients with AP CML, BP CML, or ALL, in a phase 1, open-label dose-escalation study. All patients were resistant or intolerant to imatinib. The overall CCyR rate was 30\% $(n=25)$. Whereas the responses were maintained after $2-19$ months in CP or AP CML patients, the responses of BP CML and ALL patients were 
of short duration. Mutational testing was performed in all patients, although mutations were only detected in $71 \%$ of patients at baseline. Responses were observed across all $B C R-A B L$ genotypes, with the exception of T315I (associated with resistance to both imatinib and dasatinib). Diarrhea (23\%), peripheral edema (19\%), and headache (10\%) were commonly reported AEs. Neutropenia and thrombocytopenia (grade $3 / 4$ ) were reported in $45 \%$ and $35 \%$ of CP CML patients and $89 \%$ and $80 \%$ of BP CML and ALL patients.

Cortes et $\mathrm{al}^{27}$ reported data from 74 myeloid BP (MBP) CML and 42 lymphoid BP (LBP) CML patients, who took part in two phase 2, open-label, single-arm, international studies on dasatinib (START-B and START-L), respectively. The CCyR rates at 8 months were $27 \%(n=20)$ and $43 \%$ $(\mathrm{n}=43)$ for MBP and LBP patients, respectively. After 8 months, the discontinuation rates (due to AEs) were $11 \%$ and $2 \%$ for MBP and LBP patients, respectively. Disease progression was reported for three imatinib-resistant and no imatinib-intolerant patients. Baseline $B C R-A B L$ mutation data were available from about $95 \%$ of patients. Mutations associated with very high imatinib resistance (M244V, G250E, Y253H, E255K, E255V, T315I, F359V, H396R) were associated with the lowest response rates to dasatinib. Among MBP CML patients, the most frequently reported AEs (any grade) were diarrhea (36\%), pleural effusion (28\%), peripheral edema (19\%), and dyspnea (18\%). The most common grade 3/4 AEs in MBP patients were pleural effusion (14\%), diarrhea (8\%), GI hemorrhage (8\%), and dyspnea (7\%). Diarrhea (31\%), fatigue (29\%), and nausea and vomiting $(24 \%)$ were the most common AEs (any grade) reported by LBP CML patients. The most frequent grade $3 / 4$ AE in LBP CML patients was febrile neutropenia $(12 \%)$.

Ottmann et $\mathrm{al}^{29}$ reported results from the START-L trial, focusing on ALL patients $(n=36)$. The rate of best CCyR at 8 -month follow-up was $58 \%(n=21)$. Of the $67 \%(n=15)$ of patients who had achieved a major hematologic response (MHR), five had experienced disease progression by the 8-month follow-up. In this study, the T315I mutation was found in six patients (17\%), and was, as expected, associated with a lack of response. However, overall response rates for patients with resistance mutations were comparable to those for the total population (eg, MCyR was achieved by $56 \%$ of patients with any mutation, compared with $58 \%$ of the total patient population). The most frequently reported AEs of any grade were diarrhea $(31 \%)$, pyrexia $(25 \%)$, and nausea $(22 \%)$, whereas the most common grade $3 / 4$ events were febrile neutropenia (11\%), diarrhea (8\%), and asthenia (8\%).
In the international phase 2 START-C study, $387 \mathrm{CP} C M L$ patients who were resistant $(n=288)$ or intolerant $(n=99)$ to imatinib were enrolled. Rates of CCyR and MMR after a minimum follow-up of 24 months were $53 \%$ and $47 \%$, respectively. Rates of PFS and OS at 24 months were $80 \%$ and $94 \%$, respectively. With the exception of the T315I mutation, responses were observed across all mutations. Thrombocytopenia (49\%), neutropenia (50\%), pleural effusion $(9 \%)$, dyspnea (6\%), bleeding (4\%), diarrhea (3\%), and fatigue $(3 \%)$ were among the most common grade $3 / 4$ AEs.

Guilhot et $\mathrm{al}^{26}$ recruited 107 AP CML patients (resistant or intolerant to imatinib) to an international, open-label phase 2 study. At 8-month follow-up, the CCyR rate was $24 \%$. After a minimum of 8 months of follow-up, $76 \%$ of patients were progression-free. Imatinib-resistance mutations were identified in $60 \%$ of patients tested at baseline. With the exception of T315I, the identified imatinib-resistance mutations were generally not associated with low response rates to dasatinib. Grade 3/4 hematological AEs occurred in $61 \%$ (leukopenia) to $82 \%$ (thrombocytopenia) of patients. The most frequent nonhematological AEs of any grade were diarrhea $(50 \%)$, headache $(28 \%)$, pyrexia, fatigue, and pleural effusion (23\%), and of grade $3 / 4$ were GI bleeding $(7 \%)$ and diarrhea $(6 \%)$.

\section{Nilotinib single-arm (registrational) study}

In a phase 2, open-label, international study, $318 \mathrm{CP} \mathrm{CML}$ patients intolerant or resistant to imatinib received nilotinib $400 \mathrm{mg} \mathrm{BID} .{ }^{31}$ Rates of CCyR and MCyR at 6 months were $31 \%$ and $48 \%$, respectively, and 12-month OS was estimated at $95 \%$. Baseline $B C R-A B L$ mutation status data were available for $56 \%$ of patients. Rates of MCyR and CCyR were lower in patients with mutations than in those without $(42 \%$ and $23 \%$ vs $51 \%$ and $35 \%$, respectively). T315I was the only mutation associated with MCyR and complete hematologic response (CHR) rates of $0 \%$. Rash, nausea, pruritus, fatigue, and headache were the most common AEs (all grades, $28 \%-19 \%$ ), with rash, headache, and diarrhea as the most frequent grade $3 / 4$ AEs $(3 \%, 2 \%$, and $2 \%$, respectively). In 2011, Kantarjian et $\mathrm{al}^{50}$ published 24-month follow-up data: $44 \%$ of patients achieved a cumulative CCyR (41\% of imatinib-resistant and 51\% of imatinib-intolerant patients). The median time to CCyR was approximately 3.2 months. MMR was reported in 28\% of patients (294 of 321 were evaluated). At 24 months, the estimated PFS was $64 \%$. Baseline CHR was found to be a predictive factor for achieving MCyR, CHR, MMR, and PFS. No changes in the overall AE profile were observed after 24 months. 


\section{Bosutinib single-arm study}

Bosutinib was evaluated in $288 \mathrm{CP}$ CML patients resistant or intolerant to imatinib in a phase $1 / 2$ open-label, multicenter study by Cortes et al. ${ }^{51}$ After a median follow-up period of 24.2 months, the cumulative CCyR rate was $41 \%(n=110)$, and $64 \%(n=50)$ of these patients achieved an MMR. Rates of OS were $97 \%$ and $92 \%$ at 1 and 2 years, respectively. Baseline mutation status was available for $40 \%$ of patients (42\% of imatinib-resistant and $36 \%$ of imatinib-intolerant patients). Rates of CHR and MCyR were similar for patients with and without mutations. Diarrhea (84\%), nausea (44\%), rash (44\%), and vomiting (35\%) were the most frequent nonhematological AEs. Reported grade 3/4 hematological abnormalities were thrombocytopenia (24\%), neutropenia (18\%), and anemia (13\%). On-treatment grade $3 / 4$ elevations of ALT and AST were reported by $10 \%$ and $5 \%$ of patients, respectively. Gambacorti-Passerini et al ${ }^{9}$ reported 31.8-month (median) follow-up data. The best cumulative CCyR observed was $43 \%(n=114)$. MMR was also observed in $43 \%(n=85)$ of evaluable patients. The authors estimated the OS to be $97 \%$ and $91 \%$ at 1 and 2 years, respectively. At 1 and 2 years, the estimated PFS rates were $91 \%$ and $81 \%$. Rates of CHR between $33 \%$ and $100 \%$ were reported for the different mutations identified, including one of three patients with the T315I mutation. Rates of MCyR ranged from $0 \%$ (T315I) to $75 \%$. Diarrhea, nausea, and vomiting were the most common AEs, and the AE profile was broadly similar to that previously reported for bosutinib. The study also collected HRQoL data. ${ }^{8}$ Significant improvements in five subscales were reported by imatinib-resistant patients at 12,24, and 48 weeks, exceeding the minimally important difference (MID) at 48 weeks. At 96 weeks, changes were significant for all but two subscales (imatinib-resistant patients), all but one exceeding the MID. Imatinib-intolerant patients first reported significant changes in four subscales at 24 weeks, six subscales at 48 weeks (of which the MID was exceeded for five), and similar to the imatinib-resistant patients, experienced improvement in all but two subscales, of which all but one exceeded the MID at 96 weeks (see Table 4).

\section{Nilotinib/dasatinib after imatinib}

Garg et $a l^{34}$ reported both second- and third-line results. Of the 34 patients treated with second-line nilotinib, 17 were in $\mathrm{CP}$, ten in AP, and seven in BP. The best observed CCyR and MMR rates were $9 \%(n=3)$ and $15 \%(n=5)$, respectively. In the second-line dasatinib arm, eight $\mathrm{CP}$, three $\mathrm{AP}$ and three BP patients were treated. Best responses included 14\% CCyR $(n=2)$ and no MMR. The median time on the second-line treatment was 8.3 months. Data on AEs are reported in the third-line section.

\section{Dasatinib dosing studies}

Two dasatinib dosing studies were identified. Dasatinib $140 \mathrm{mg}$ OD was compared with $70 \mathrm{mg}$ BID in an open-label phase 3 trial, with results for two separate populations reported..$^{22,52}$ Enrolling 317 AP patients resistant or intolerant to imatinib, Kantariian et $\mathrm{al}^{22}$ reported results after a minimum of 0.16 months and a median of 15 months of follow-up. The CCyR rates were 32\% with the OD and 33\% with the BID administration schedule, respectively (MMR rates were not reported). At 24 months, estimated PFS rates were $51 \%$ and $55 \%$ with the OD and BID administration schedules, respectively. The administration schedule did not appear to affect the response rate by mutation status. The most common AEs were diarrhea, fluid retention, nausea, headache, and fatigue. The incidence of GI bleeding and fluid-retention events was lower with the OD administration schedule. Saglio et $\mathrm{al}^{52}$ reported results for 149 patients in myeloid and 61 patients in LBP. Both groups were randomized $1: 1$ to dasatinib $140 \mathrm{mg}$ OD or $70 \mathrm{mg}$ BID. In MBP patients, the CCyR rates were $14 \%$ with the $\mathrm{OD}$ and $21 \%$ with the BID administration schedule. In LBP patients, the CCyR rates were higher, with $38 \%$ and $36 \%$ for OD and BID administration schedules, respectively (no MMR rates reported). The 24-month PFS rate was $11 \%$ for the OD and $18 \%$ for the BID administration schedule. Rates of MHR were similar for patients with or without baseline mutations for both dosing schedules, except for patients with the T315I mutation, none of whom achieved an MHR. Safety results were generally consistent with those reported by Kantarjian et al $;{ }^{22}$ only pleural effusion was less frequent with the OD regimen versus BID administration in LBP but not MBP patients.

Shah et $\mathrm{al}^{23}$ randomized 670 patients to dasatinib $100 \mathrm{mg}$ OD, $50 \mathrm{mg}$ BID, $140 \mathrm{mg}$ OD, or $70 \mathrm{mg}$ BID in an open-label phase 3 trial. The minimum and median follow-ups at the time of the analysis were 6 and 8 months, respectively. No major differences in response rates were observed: CCyR rates were $41 \%$ with $100 \mathrm{mg}$ OD, $42 \%$ with $50 \mathrm{mg}$ BID, $44 \%$ with $140 \mathrm{mg}$ OD, and $45 \%$ with $70 \mathrm{mg}$ BID. Rates of MMR were not reported. Rates of disease progression or death were $8 \%$ with $100 \mathrm{mg}$ OD, $50 \mathrm{mg}$ BID, and $140 \mathrm{mg}$ $\mathrm{OD}$, and $11 \%$ with $70 \mathrm{mg}$ BID. The dose/administration schedule did not appear to affect the response rate by mutation status. Patients in the 100-mg OD treatment arm experienced fewest treatment-related AEs (eg, pleural effusion, thrombocytopenia, or nausea). Two-year follow-up results were reported in subsequent publications. ${ }^{53,54}$ The observed 
Table 4 QoL data

\begin{tabular}{|c|c|c|c|c|}
\hline \multirow{2}{*}{\multicolumn{2}{|c|}{$\begin{array}{lc}\text { Study } & \text { Publication } \\
\text { First-line RCT - bosutinib versus imatin }\end{array}$}} & \multicolumn{2}{|c|}{ QoL outcomes } & \\
\hline & & & & \\
\hline \multirow[t]{10}{*}{ BELA } & Lipton et al ${ }^{49}$ & Mean (SD) & Bosutinib $(n=237)$ & Imatinib $(n=24 I)$ \\
\hline & & Baseline & & \\
\hline & & FACT-G & $83.8(12.0)$ & $83.5(14.9)$ \\
\hline & & FACT-Leu & $137.8(\mid 8.6)$ & $136.4(23.0)$ \\
\hline & & Month 3 & & \\
\hline & & FACT-G & 83.5 (I4.2); $\Delta 0.4$ (I I.3) & 84.1 (I6.3); $\Delta 0.6(10.7)$ \\
\hline & & FACT-Leu & I38.I (20.7); $\Delta 0.3$ (I6.I) & I $39.0(24.2) ; \Delta 2.4(16.7)$ \\
\hline & & Month I2 & & \\
\hline & & FACT-G & 83.9 (I4.I), $\Delta 0.1$ (I2.I) & 84.5 (I7.I); $\Delta \mathrm{I} . \mathrm{I}(\mathrm{I} 3.0)$ \\
\hline & & FACT-Leu & I 38.4 (22.2); $\Delta 0.5$ (19.1) & I $40.3(23.9) ; \Delta 3.5(\mid 8.8)$ \\
\hline \multicolumn{5}{|c|}{ Second-line bosutinib study } \\
\hline \multirow[t]{13}{*}{ NCT00261846 } & Trask et $\mathrm{al}^{8}$ & Mean (SD) & Imatinib-intolerant & Imatinib-resistant \\
\hline & & Baseline & & \\
\hline & & FACT-G & $79.1(16.8)$ & $82.2(14.4)$ \\
\hline & & FACT-Leu & $130.3(24.6)$ & $134.8(21.6)$ \\
\hline & & Week 12 & & \\
\hline & & FACT-G & $\Delta 0.1$ & $\Delta \mathrm{l} . \mathrm{l}$ \\
\hline & & FACT-Leu & $\Delta \mathrm{I} .7$ & $\Delta 3.1$ \\
\hline & & Week 48 & & \\
\hline & & FACT-G & $\Delta 5.8$ & $\Delta \mathrm{l} . \mathrm{I}$ \\
\hline & & FACT-Leu & $\Delta 9.6$ & $\Delta 3.2$ \\
\hline & & Week 96 & & \\
\hline & & FACT-G & $\Delta 5.2$ & $\Delta \mathrm{I} .2$ \\
\hline & & FACT-Leu & $\Delta 9.3$ & $\Delta 4.3$ \\
\hline
\end{tabular}

CCyR rates were 54\% with $50 \mathrm{mg}$ BID, and 50\% with each of the other three administration schedules; MMR rates were $37 \%$ with $100 \mathrm{mg} \mathrm{OD}$, and 38\% with each of the other three administration schedules. Response rates for patients with any or no mutations were comparable between the treatment groups (except for T315I, which was associated with no CCyR). The differences between the AE profiles of the different dose/administration schedules were consistent with those reported earlier. In 2010, Shah et al ${ }^{55}$ presented 4-year follow-up results, reporting that response rates were similar in all treatment arms. Results were presented for the 100-mg OD arm only, ${ }^{56}$ as the percentage remaining on treatment was highest in this arm (35\%, compared with $31 \%$ on $50 \mathrm{mg}$ BID or on $70 \mathrm{mg}$ BID, and $27 \%$ on $140 \mathrm{mg}$ OD). The best overall response within 24 months was 50\% CCyR. Within 5 years, the cumulative MMR rate was $44 \%$, and $5 \%(n=8)$ of patients had experienced transformation. It was reported that nonhematological and hematological AEs first occurred generally within 24 and 12 months of treatment.

\section{High-dose imatinib versus dasatinib}

in extensively pretreated patients

In a study by Kantarjian et $\mathrm{al}^{21} 150$ patients who were resistant to imatinib were randomized $(2: 1)$ to either dasatinib
$140 \mathrm{mg}$ or imatinib $800 \mathrm{mg}$. Patients had undergone previous treatments for between 6 and 166 months. Crossover was permitted upon progression, lack of response, or intolerance. After 12 weeks of randomized treatment, CCyR rates with dasatinib were significantly higher than those with high-dose imatinib ( $22 \%$ vs $8 \%, P=0.041)$. After a median follow-up of 15 months, the superiority of dasatinib was maintained, with CCyR rates of $40 \%$ (dasatinib) and 16\% (imatinib, $P=0.004$ ); MMR rates also favored dasatinib (16\%) over imatinib $(4 \%, P=0.038)$. In total, $15 \%$ of patients crossed from the dasatinib group to the imatinib group, and $80 \%$ of patients randomized to imatinib crossed over to dasatinib. At baseline, 52 patients (38\%) had an imatinib-resistant $B C R-A B L$ mutation. Of these, 19 of 41 patients (46\%) in the dasatinib group and three of 11 patients (27\%) on imatinib achieved an MCyR $(P=0.282)$. The observed AEs corresponded to the known safety profile of the treatments, and there was no major difference between treatments. Two-year follow-up data indicated that favorable response rates were maintained with dasatinib. ${ }^{24}$ The CCyR was $44 \%$ with dasatinib versus $18 \%$ with imatinib $(P=0.0025)$, and the MMR rates were $29 \%$ with dasatinib versus $12 \%$ with imatinib $(P=0.028)$. Compared with imatinib, dasatinib-treated patients reported 
a higher incidence of grade 3/4 neutropenia, thrombocytopenia, and leukopenia.

\section{Third-line treatments}

Bosutinib $^{10}$ and nilotinib ${ }^{32}$ have been investigated as thirdline treatment options in single-arm studies, whereas two studies allowed a choice of either nilotinib or dasatinib as treatment. ${ }^{33,34}$ An overview of the included publications, including patient baseline characteristics and main efficacy outcomes, is provided in Tables 2 and 3.

\section{Nilotinib}

Patients with CML in CP $(n=37)$ or AP $(n=17)$ were enrolled in an international phase 2 study by Giles et al. ${ }^{32}$ After a median follow-up of 12 months, no patients in AP and $24 \%(n=9)$ of patients in CP achieved CCyR. At 18 months, the PFS was estimated to be $59 \%$, with a survival rate of $86 \%$; median OS was not reached at that point. Disease progression $(\mathrm{n}=19,35 \%)$, AEs $(\mathrm{n}=10,19 \%)$, and death $(\mathrm{n}=2,4 \%)$ were the most common reasons for discontinuation. The most common grade 3/4 AEs were neutropenia (23\% CP, $33 \%$ AP), thrombocytopenia (28\% CP, 19\% AP), hyperphosphatemia (13\% CP, 24\% AP), and elevated lipase levels (25\% CP, 10\% AP).

\section{Nilotinib/dasatinib}

Ibrahim et $\mathrm{al}^{33}$ and Garg et $\mathrm{al}^{34}$ evaluated $26 \mathrm{CP}$ CML patients and $48 \mathrm{CML}$ patients (25 in CP, 10 in AP, 13 in BP) who had failed on imatinib therapy as well as on dasatinib or nilotinib. The third-line study treatment was nilotinib or dasatinib in both studies. In the study by Ibrahim et al, ${ }^{33}$ cumulative rates of CCyR and MMR after a median of 21.5 months' follow-up were $34.6 \%(n=9)$ and $19.2 \%(n=5)$, respectively. Probabilities of EFS and OS at 30 months were $45.7 \%$ and $46.7 \%$, respectively. AEs were not reported. The authors found that previous achievement of a cytogenetic response was a predictor for third-line treatment success as well as OS.

During third-line treatment in the study by Garg et al, ${ }^{34}$ CCyR was achieved by five patients (31\%) in CP, two (25\%) in AP, and two (20\%) in BP in patients receiving dasatinib third-line. In the nilotinib group, one (11\%) patient in $\mathrm{CP}$, no patients in AP, and one (33\%) in BP achieved CCyR. The corresponding numbers of patients reaching MMR were two $(13 \%)$ in $\mathrm{CP}$, one $(13 \%)$ in $\mathrm{AP}$, and one $(10 \%)$ in $\mathrm{BP}$ in patients receiving dasatinib, and three $(33 \%)$ in $\mathrm{CP}$, one $(50 \%)$ in AP, and no patients in BP in nilotinib-treated patients. AEs were not reported. The median EFS was 13 months overall, ranging from 20 months for CP patients, over 5 months for AP patients, and only 3 months for BP patients.

\section{Bosutinib}

In a phase $1 / 2$, open-label, multicenter study, ${ }^{57}$ bosutinib $500 \mathrm{mg} /$ day was evaluated in CP CML patients in the thirdline setting. Of the 118 enrolled patients, 64 were resistant to prior imatinib and either dasatinib $(n=37)$ or nilotinib ( $n=27), 50$ were intolerant to prior imatinib and dasatinib, and four received fourth-line treatment (having received prior imatinib, nilotinib, and dasatinib). After a median follow-up time of 28.5 months (ranging from 20.0 months in the dasatinib-resistant group to 34.5 months in the dasatinib-intolerant group), the best cumulative CCyR rate in the overall study population was $24 \%(\mathrm{n}=26)$. Within the separate cohorts, the reported CCyR rates were $14 \%(n=5)$ and $27 \%(n=7)$ in the dasatinib- and nilotinib-resistant groups, respectively, $28 \%(\mathrm{n}=12)$ in the dasatinib-intolerant group, and $50 \%$ $(\mathrm{n}=2)$ in the fourth-line patients. Estimated OS was 91\% at 1 year and $83 \%$ at 2 years. Within the separate cohorts, the estimated 2-year OS was $75 \%$ and $92 \%$ in the dasatinib- and nilotinib-resistant groups, respectively, $85 \%$ in the dasatinibintolerant group and $75 \%$ in the fourth-line patients. Five patients (three dasatinib-resistant, one nilotinib-resistant, and one fourth-line) progressed to AP CML. The most common AEs were GI-related. The most common grade 3/4 AEs were thrombocytopenia $(25 \%, \mathrm{n}=30)$, neutropenia $(19 \%, \mathrm{n}=23)$, hypermagnesemia $(12 \%, \mathrm{n}=14)$, diarrhea $(8 \%, \mathrm{n}=10)$, and elevated ALT $(7 \%, \mathrm{n}=8)$.

\section{Discussion}

This qualitative review is limited by the small number of trials investigating any given drug or combination treatment. Initially, the second-generation TKIs were investigated in the second- and third-line setting, as there were no active comparators available to be used in clinical trials and a license in this setting could therefore be obtained on the basis of a single-arm trial.

The structured literature search, including conference abstracts, and the assessment of the methodological quality of the included articles by two individuals separately, contribute to the strength of evidence provided by this systematic review.

\section{First-line treatments}

First-generation TKIs represent the first targeted therapy for CML, superseding chemotherapeutic agents and IFN- $\alpha$. The comparison of imatinib with IFN- $\alpha$ (plus cytarabine) 
in a key trial (IRIS), follow-up data which is reported up to 8 years, demonstrated the clear superiority of imatinib over IFN- $\alpha{ }^{14,35-39}$ Imatinib was the first TKI to be extensively used for the treatment of CML. The two included dose-finding studies $^{18,19,48}$ did not report a difference between $400 \mathrm{mg} /$ day and $800 \mathrm{mg} /$ day in CCyR rates at 1 year, although results up to 24 months favored the higher dose. ${ }^{18,19}$

When second-generation TKIs became available, they were naturally compared with imatinib. Dasatinib was found to result in significantly higher cumulative response rates by 12 and 18 months than imatinib in the single trial comparing the treatments. ${ }^{15,42}$ At 24-month follow-up, CCyR, MMR, and CMR were higher in the dasatinib treatment group compared with imatinib, ${ }^{45}$ although the difference was no longer statistically significant for CCyR. Nilotinib was also associated with higher response rates reported at 12, 18, and 24 months, ${ }^{17,44,46}$ compared with imatinib in a single trial. At 12 and 24 months, CCyR, MMR, and CMR rates were significantly higher in both nilotinib treatment groups than in the imatinib group $(P<0.001)$. With bosutinib, ${ }^{11,12} \mathrm{CCyR}$ rates at 1 year were numerically higher than those in the imatinib group, whereas MMR rates at and by 1 year were significantly higher with bosutinib than with imatinib $(P=0.002$ and $P<0.001$, respectively). With bosutinib, responses were also achieved significantly faster $(P<0.001)$. Superior rates of MMR, CMR, and similar rates of CCyR were reported for bosutinib compared with imatinib at 24 months of follow-up. ${ }^{47}$ QoL data from the bosutinib study were published, ${ }^{49}$ indicating that after a minimum follow-up period of 12 months (median 16.6 months), no significant between-group difference was reported between the bosutinib and imatinib treatment arms.

Therefore, although relatively few RCTs have been published to date, available results indicate that treatment with the second-generation TKIs is associated with higher response rates for most outcomes at 2-year follow-up compared with imatinib. ${ }^{43,46,47}$ There are also indications that a faster response can be achieved with second-generation TKIs, ${ }^{12}$ and there is emerging evidence that a quicker response may be associated with a more favorable outcome for imatinib. ${ }^{58}$ All second-generation TKIs reported higher rates of CMR ( $\leq 4.5-\log$ reduction) by 24 months compared with imatinib. Achievement of a "deeper" response appears to be clinically relevant, as indicated by results from the Stop Imatinib (STIM) study, ${ }^{59}$ in which imatinib treatment was stopped in 100 patients who had been on the drug for at least 2 years and who had achieved CMR during treatment. After stopping imatinib treatment, $41 \%$ of patients maintained CMR at 1-year follow up, suggestive of a TKI-induced "cure" in a subset of patients.

In the absence of head-to-head RCTs comparing the second-generation TKIs, it is challenging to make robust conclusions on their relative efficacy. A recent indirect comparison reported on the relative efficacy of nilotinib and dasatinib and concluded that patients treated with nilotinib 300 mg BID experienced significantly higher MMR by 12 months compared with dasatinib-treated patients. ${ }^{60}$

\section{Second-line treatments}

The second-line single-arm studies on dasatinib, nilotinib, and bosutinib showed that these agents can elicit responses (CyRs, HRs, and MRs) in patients who are resistant or intolerant to imatinib. Indeed, results from the studies conducted for nilotinib and dasatinib resulted in these agents being granted a license for this indication. Recently published data for bosutinib are also encouraging, and it is currently undergoing regulatory review in several countries. In contrast to the included RCTs (regardless of first- or second-line), which enrolled only CP CML patients, four of the five registrational single-arm studies on dasatinib focused on patients in BP, AP, or mixed-patient populations. In the course of the bosutinib single-arm study, ${ }^{9,51}$ QoL data were collected ${ }^{8}$ with a statistically significant and clinically meaningful increase reported at weeks 36, 48, and 96 follow-up.

\section{Third-line treatments}

No RCTs on third-line treatments were eligible for inclusion in this systematic review. This is primarily a result of the small numbers of eligible patients who are resistant/intolerant to multiple TKIs and therefore available for enrollment into a study and the paucity of active comparators in this setting. However, four single-arm studies were identified. Garg et al, ${ }^{34}$ who followed 48 patients treated successively with three TKIs (starting with imatinib, followed by dasatinib and nilotinib second- or third-line), found that while a response was induced in some patients, it was not durable. Nilotinib was also found to be efficacious as a third-line TKI treatment. ${ }^{32}$ Another similar study on third-line TKIs concluded that while therapy was only efficacious in a small proportion of patients, prior CCyR on first- or second-line TKI treatment could serve as a predictor for CCyR to thirdline TKI treatment. ${ }^{33}$ The largest study to date has been conducted with bosutinib, ${ }^{57}$ and demonstrated clinical activity (comparable with other second-generation TKIs) with an acceptable AE profile. 
Table 5 Incidence (percentage) of adverse events reported in included studies (all grade and grade 3/4)

\begin{tabular}{|c|c|c|c|c|}
\hline \multirow[t]{2}{*}{ Adverse event } & \multicolumn{4}{|c|}{$\%$ of patients with adverse event; (no of patients); (follow-up) } \\
\hline & $\begin{array}{l}\text { Imatinib, } 400 \mathrm{mg} \text { OD } \\
\text { up to } 400 \mathrm{mg} \mathrm{BD}\end{array}$ & $\begin{array}{l}\text { Dasatinib, } \\
\text { I } 00 \text { mg OD }\end{array}$ & $\begin{array}{l}\text { Nilotinib, } \\
400 \text { mg BD }\end{array}$ & $\begin{array}{l}\text { Bosutinib } \\
500 \mathrm{mg} \text { OD, }\end{array}$ \\
\hline \multicolumn{5}{|l|}{ First-line RCTs } \\
\hline Incidence of neutropenia: number of studies & 5 (I589) (I2-24 months) & I (260) (24 months) & I (28I) (24 months) & I (250) (I 8 months) \\
\hline Incidence of neutropenia: all grades & $22-68(610)$ & $11(260)$ & $38(28 \mathrm{I})$ & NR \\
\hline Incidence of neutropenia: grade $3 / 4$ & $7-24(17 \mid 2)$ & $21(260)$ & $10(28 \mathrm{I})$ & II (250) \\
\hline $\begin{array}{l}\text { Incidence of thrombocytopenia: number } \\
\text { of studies }\end{array}$ & $6(1712)$ (12-24 months) & 2 (383) (24 months) & I (28I) (24 months) & I (250) (I8 months) \\
\hline Incidence of thrombocytopenia: all grades & $8-56(610)$ & NR & $12(28 \mid)$ & NR \\
\hline Incidence of thrombocytopenia: grade $3 / 4$ & $8-18(1720)$ & $18-20(383)$ & $40(281)$ & II (250) \\
\hline Incidence of diarrhea: number of studies & 5 (I589) (I2-24 months) & I (260) (24 months) & I (28I) (24 months) & I (250) (I8 months) \\
\hline Incidence of diarrhea: all grades & $0-69(1113)$ & $21(260)$ & $6(28 I)$ & $69(250)$ \\
\hline Incidence of diarrhea: grade $3 / 4$ & $0-2 \mid(\mid 338)$ & NR & $0(28 I)$ & II (250) \\
\hline Incidence of vomiting: number of studies & 4 (II29) (I2-24 months) & I (260) (24 months) & I (28I) (24 months) & I (250) (I8 months) \\
\hline Incidence of vomiting: all grades & $4-32(1113)$ & $5(260)$ & $\mathrm{I}(28 \mathrm{I})$ & $32(250)$ \\
\hline Incidence of vomiting: grade $3 / 4$ & $0-2(862)$ & NR & $9(28 I)$ & $3(250)$ \\
\hline Incidence of nausea: number of studies & 4 (III3) (I2-24 months) & I (260) (24 months) & I (28I) (24 months) & I (250) (I8 months) \\
\hline Incidence of nausea: all grades & $11-35(1113)$ & $10(260)$ & $19(28 \mathrm{I})$ & $31(250)$ \\
\hline Incidence of nausea: grade $3 / 4$ & $0-I(862)$ & NR & $\mathrm{I}(28 \mathrm{I})$ & $I(250)$ \\
\hline Incidence of rash: number of studies & 5 (I589) (12-24 months) & I (260) (24 months) & I (28I) (24 months) & I (250) (I8 months) \\
\hline Incidence of nausea: all grades & $\mathrm{I}-22(\mathrm{I} 12 \mathrm{I})$ & II (260) & $\mathrm{II}(28 \mathrm{I})$ & $22(250)$ \\
\hline Incidence of nausea: grade 3/4 & $1-36(1589)$ & NR & $\mathrm{I}(28 \mathrm{I})$ & $2(250)$ \\
\hline $\begin{array}{l}\text { Treatment discontinuations due to AEs: } \\
\text { number of studies }\end{array}$ & 5 (1393) (I2-24 months) & I (260) (24 months) & I (28I) (24 months) & I (250) (I8 months) \\
\hline Treatment discontinuations due to AEs & $4.5-12(1393)$ & $7(260)$ & $12(28 \mathrm{I})$ & $23(250)$ \\
\hline
\end{tabular}

\section{Toxicity profiles}

Each of the TKIs is associated with a characteristic AE profile (Table 5). Treatment with imatinib is predominantly accompanied by superficial edema, nausea, muscle cramps, and elevated rates of some hematological AEs (neutropenia and hypophosphatemia). Considering the second-generation TKIs, treatment with nilotinib is associated with increased incidence of rash, dasatinib with certain hematological AEs, and fluid retention (including pleural effusion), and bosutinib-treated patients report increased GI AEs.

\section{Conclusion}

There are a number of findings from the present systematic review. Firstly, there is now a wealth of data available over a long follow-up period (up to 8 years) to indicate that imatinib is clinically superior to IFN plus cytarabine, ${ }^{14}$ and that the efficacy of imatinib is not improved by the addition of IFN- $\alpha$, cytarabine, or granulocyte-macrophage colony-stimulating factor. Secondly, despite relatively short follow-up ( 2 years), there is increasing evidence to indicate that treatment with the second-generation TKIs dasatinib, nilotinib, and bosutinib offers improved, "deeper" responses that are achieved more rapidly compared with standard-dose imatinib in CP CML patients in both the first- and secondline setting. Although each of these therapies is associated with a distinct AE profile, the majority of AEs are low-grade and manageable. However, longer follow-up is required to confirm that the improved efficacy of the second-generation TKIs is maintained and to allow robust conclusions with regard to the effect of these improved response rates on OS. Although outside the scope of the current review, there are several therapies currently under investigation. In particular, ponatinib, a TKI inhibitor active against the $B C R-A B L$ gene, is a promising agent. The single-arm, phase 2 Ponatinib $\mathrm{Ph}+$ ALL and CML Evaluation (PACE) trial ${ }^{61}$ was conducted in 397 patients with refractory CML in CP, AP, or $\mathrm{BP}$, or $\mathrm{Ph}+$ ALL, resistant or intolerant to dasatinib or nilotinib, or with the resistant T315I mutation. Initial data at a median follow-up of 57 days suggest that ponatinib has activity in heavily pretreated patients and in patients with the T315I mutation.

The current availability of several second-generation TKIs should allow selection of the most relevant treatment on an individualized basis, taking into account any comorbid conditions and mutation status if known. Currently, 
there is a paucity of data reporting on the effects of treatment on QoL outcomes (of importance in the management of CML, which requires long-term therapy) and in the third-line setting, where patients currently have limited treatment options. Further studies are required to address both these issues.

\section{Acknowledgment}

The authors would like to thank Dr David Marin for his work on reviewing our manuscript.

\section{Disclosure}

Stephen Mitchell and Sarah Batson are employees of Abacus International who were paid consultants to Pfizer in connection with development of this manuscript. All other authors have no conflicts of interest to disclose.

\section{References}

1. Heisterkamp N, Stam K, Groffen J, de Klein A, Grosveld G. Structural organization of the $b c r$ gene and its role in the $\mathrm{Ph}$ 'translocation. Nature. 1985;315(6022):758-761.

2. Rowley JD. A new consistent chromosomal abnormality in chronic myelogenous leukaemia identified by quinacrine fluorescence and Giemsa staining. Nature. 1973;243(5405):290-293.

3. Penserga ET, Skorski T. Fusion tyrosine kinases: a result and cause of genomic instability. Oncogene. 2007;26(1):11-20.

4. Rohrbacher M, Hasford J. Epidemiology of chronic myeloid leukaemia (CML). Best Pract Res Clin Haematol. 2009;22(3):295-302.

5. Kantarjian H, Faderl S, Talpaz M. Chronic myelogenous leukemia. In: DeVita, Hellman, Rosenberg, editors. Cancer: Principles and Practice of Oncology. Philadelphia: Lippincott Williams \& Wilkins; 2001.

6. DeVita V, Lawrence T, Rosenberg S. DeVita, Hellman, and Rosenberg's Cancer: Principles and Practice of Oncology. 8th ed. Philadelphia: Lippincott Williams \& Wilkins; 2008.

7. Pavlovsky C, Kantarjian H, Cortes JE. First-line therapy for chronic myeloid leukemia: past, present, and future. Am J Hematol. 2009;84(5):287-293.

8. Trask PC, Cella D, Besson N, Kelly V, Masszi T, Kim DW. Health-related quality of life of bosutinib (SKI-606) in imatinibresistant or imatinib-intolerant chronic phase chronic myeloid leukemia. Leuk Res. 2012;36(4):438-442.

9. Gambacorti-Passerini C, Kim DW, Turkina A, et al. Bosutinib (SKI-606) as second-line therapy for chronic phase chronic myeloid leukemia following imatinib failure: analyses of cross-intolerance and response by prior response to imatinib. 16th Congress of the European Hematology Association; June 9-12, 2011; London, UK.

10. Gambacorti-Passerini C, Brümmendorf T, Cortes J, et al. Bosutinib as third-line therapy for chronic phase chronic myeloid leukemia following failure with imatinib and dasatinib or nilotinib. 16th Congress of the European Hematology Association; June 9-12, 2011; London, UK.

11. Gambacorti-Passerini C, Kim DW, Kantarjian H, et al. An ongoing phase 3 study of bosutinib (SKI-606) versus imatinib in patients with newly diagnosed chronic phase chronic myeloid leukemia. Abstract 208. 52nd American Society of Hematology Annual Meeting; December 10-13, 2010; Orlando, FL.

12. Gambacorti-Passerini C, Cortes J, Khoury HJ, et al. Bosutinib (BOS) versus imatinib (IM) in patients with chronic phase chronic myeloid leukemia (CP CML) in the BELA trial: 18-month follow-up. Abstract 6509. American Society of Clinical Oncology Annual Meeting; June 3-7, 2011; Chicago, IL.
13. The Cochrane Collaboration. Cochrane Handbook for Systematic Reviews of Interventions. Version 5.1.0. 2011. Available from: http://www.cochrane-handbook.org. Accessed June 26, 2012.

14. O'Brien SG, Guilhot F, Larson RA, et al. Imatinib compared with interferon and low-dose cytarabine for newly diagnosed chronic-phase chronic myeloid leukemia. N Engl J Med. 2003;348(11):994-1004.

15. Kantarjian H, Shah NP, Hochhaus A, et al. Dasatinib versus imatinib in newly diagnosed chronic-phase chronic myeloid leukemia. $N$ Engl J Med. 2010;362(24):2260-2270.

16. Radich JP, Kopecky KJ, Kamel-Reid S, et al. A randomized phase II trial of dasatinib $100 \mathrm{mg}$ vs imatinib $400 \mathrm{mg}$ in newly diagnosed chronic myeloid leukemia in chronic phase (CML-CP): the S0325 Intergroup Trial. 52nd American Society of Hematology Annual Meeting and Exposition; December 4-7, 2010; Orlando, FL.

17. Saglio G, Kim DW, Issaragrisil S, et al. Nilotinib versus imatinib for newly diagnosed chronic myeloid leukemia. $N$ Engl J Med. 2010;362(24):2251-2259.

18. Cortes JE, Baccarani M, Guilhot F, et al. Phase III, randomized, open-label study of daily imatinib mesylate $400 \mathrm{mg}$ versus $800 \mathrm{mg}$ in patients with newly diagnosed, previously untreated chronic myeloid leukemia in chronic phase using molecular end points: tyrosine kinase inhibitor optimization and selectivity study. J Clin Oncol. 2010;28(3):424-430.

19. Baccarani M, Druker B, Cortes J, et al. 24 months update of the TOPS study: a phase iii, randomized open label study of $400 \mathrm{mg} / \mathrm{d}$ (SD-IM) versus $800 \mathrm{mg} / \mathrm{d}$ (HD-IM) of imatinib mesylate (IM) in patients (Pts) with newly diagnosed, previously treated chronic myeloid leukemia in chronic phase (CML-CP). Abstract 337. 51st American Society of Hematology Annual Meeting and Exposition; December 5-8, 2009; New Orleans, LA.

20. Cortes JE, Jones D, O'Brien S, et al. Results of dasatinib therapy in patients with early chronic-phase chronic myeloid leukemia. J Clin Oncol. 2010;28(3):398-404.

21. Kantarjian H, Pasquini R, Hamerschlak N, et al. Dasatinib or highdose imatinib for chronic-phase chronic myeloid leukemia after failure of first-line imatinib: a randomized phase 2 trial. Blood. 2007;109(12):5143-5150.

22. Kantarjian H, Cortes J, Kim DW, et al. Phase 3 study of dasatinib $140 \mathrm{mg}$ once daily versus $70 \mathrm{mg}$ twice daily in patients with chronic myeloid leukemia in accelerated phase resistant or intolerant to imatinib: 15-month median follow-up. Blood. 2009;113(25): 6322-6329.

23. Shah NP, Kantarjian HM, Kim DW, et al. Intermittent target inhibition with dasatinib $100 \mathrm{mg}$ once daily preserves efficacy and improves tolerability in imatinib-resistant and -intolerant chronicphase chronic myeloid leukemia. J Clin Oncol. 2008;26(19): 3204-3212.

24. Kantarjian H, Pasquini R, Levy V, et al. Dasatinib or high-dose imatinib for chronic-phase chronic myeloid leukemia resistant to imatinib at a dose of 400 to 600 milligrams daily: two-year follow-up of a randomized phase 2 study (START-R). Cancer. 2009;115(18): 4136-4147.

25. Hochhaus A, Kantarjian HM, Baccarani M, et al. Dasatinib induces notable hematologic and cytogenetic responses in chronic-phase chronic myeloid leukemia after failure of imatinib therapy. Blood. 2007;109(6):2303-2309.

26. Guilhot F, Apperley J, Kim DW, et al. Dasatinib induces significant hematologic and cytogenetic responses in patients with imatinibresistant or -intolerant chronic myeloid leukemia in accelerated phase. Blood. 2007;109(10):4143-4150.

27. Cortes J, Rousselot P, Kim DW, et al. Dasatinib induces complete hematologic and cytogenetic responses in patients with imatinibresistant or -intolerant chronic myeloid leukemia in blast crisis. Blood. 2007;109(8):3207-3213.

28. Talpaz M, Shah NP, Kantarjian H, et al. Dasatinib in imatinibresistant Philadelphia chromosome-positive leukemias. N Engl J Med. 2006;354(24):2531-2541. 
29. Ottmann O, Dombret H, Martinelli G, et al. Dasatinib induces rapid hematologic and cytogenetic responses in adult patients with Philadelphia chromosome positive acute lymphoblastic leukemia with resistance or intolerance to imatinib: interim results of a phase 2 study. Blood. 2007;110(7):2309-2315.

30. Mauro MJ, Baccarani M, Cervantes F, et al. Dasatinib 2-year efficacy in patients with chronic-phase chronic myelogenous leukemia (CML-CP) with resistance or intolerance to imatinib (START-C). J Clin Oncol. 2008;26(May 20 Suppl):Abstr 7009.

31. Kantarjian HM, Giles F, Gattermann N, et al. Nilotinib (formerly AMN107), a highly selective BCR-ABL tyrosine kinase inhibitor, is effective in patients with Philadelphia chromosome-positive chronic myelogenous leukemia in chronic phase following imatinib resistance and intolerance. Blood. 2007;110(10):3540-3546.

32. Giles FJ, Abruzzese E, Rosti G, et al. Nilotinib is active in chronic and accelerated phase chronic myeloid leukemia following failure of imatinib and dasatinib therapy. Leukemia. 2010;24(7): 1299-1301.

33. Ibrahim AR, Paliompeis C, Bua M, et al. Efficacy of tyrosine kinase inhibitors (TKIs) as third-line therapy in patients with chronic myeloid leukemia in chronic phase who have failed 2 prior lines of TKI therapy. Blood. 2010;116(25):5497-5500.

34. Garg RJ, Kantarjian H, O'Brien S, et al. The use of nilotinib or dasatinib after failure to 2 prior tyrosine kinase inhibitors: long-term follow-up Blood. 2009;114(20):4361-4368.

35. Kantarjian HM, Larson RA, Guilhot F, et al. Efficacy of imatinib dose escalation in patients with chronic myeloid leukemia in chronic phase. Cancer. 2009;115(3):551-560.

36. Druker BJ, Guilhot F, O'Brien SG, et al. Five-year follow-up of patients receiving imatinib for chronic myeloid leukemia. $N$ Engl $J$ Med. 2006;355(23):2408-2417.

37. Hochhaus A, O’Brien SG, Guilhot F, et al. Six-year follow-up of patients receiving imatinib for the first-line treatment of chronic myeloid leukemia. Leukemia. 2009;23(6):1054-1061.

38. O'Brien S, Guilhot F, Goldman J, et al. International randomized study of interferon versus ST1571 (IRIS) 7-year follow-up: sustained survival, low rate of transformation and increased rate of major molecular response (MMR) in patients (PTS) with newly diagnosed chronic myeloid leukemia in chronic phase (CML-CP) treated with imatinib (IM). Abstract 186. 50th American Society of Hematology Annual Meeting and Exposition; Decemeber 6-9, 2008; San Francisco, CA.

39. Deininger M, O'Brien S, Guilhot F, et al. International randomized study of interferon vs ST1571 (IRIS) 8-year follow up: sustained survival and low risk for progression or events in patients with newly diagnosed chronic myeloid leukemia in chronic phase (CML-CP) treated with imatinib. Abstract 1126.51st American Society of Hematology Annual Meeting and Exposition; Decemeber 5-8, 2009; New Orleans, LA.

40. Hughes TP, Hochhaus A, Branford S, et al. Long-term prognostic significance of early molecular response to imatinib in newly diagnosed chronic myeloid leukemia: an analysis from the International Randomized Study of Interferon and STI571 (IRIS). Blood. 2010;116(19):3758-3765.

41. Guilhot F, Larson J, O’Brien SG, et al. Time to complete cytogenetic response (CCyR) does not affect long-term outcomes for patients on imatinib therapy. 49th American Society of Hematology Annual Meeting and Exposition; Decemeber 8-11, 2007; Atlanta, GA.

42. Shah N, Kantarjian H, Hochhaus A, Cortes J, Bradley-Garelik MB, Zhu C, et al. Dasatinib versus imatinib in patients with newly diagnosed chronic myeloid leukemia in chronic phase (CML-CP) in the DASISION trial: 18 month follow-up. Abstract 206. 52nd American Society of Hematology Annual Meeting; Decemeber 4-7, 2010; Orlando, FL.

43. Kantarjian HM, Shah NP, Cortes JE, et al. Dasatinib or imatinib in newly diagnosed chronic phase chronic myeloid leukemia: 2-year follow-up from a randomized phase 3 trial (DASISION). Blood. 2012;119(5):1123-1129.
44. Hughes T, Hochhaus A, Saglio G, et al. ENESTnd update: continued superiority of nilotinib versus imatinib in patients with newly diagnosed chronic myeloid leukemia in chronic phase (CML-CP). 52nd American Society of Hematology Annual Meeting; December 4-7, 2010; Orlando, FL.

45. Hughes P, Hochhaus A, Saglio G, et al. ENESTnd 24-month update: continued superiority of nilotinib versus imatinib in patients with newly diagnosed chronic myeloid leukemia in chronic phase (CML-CP). Blood. 2010;116: Abstr 207.

46. Kantarjian HM, Hochhaus A, Saglio G, et al. Nilotinib versus imatinib for the treatment of patients with newly diagnosed chronic phase, Philadelphia chromosome-positive, chronic myeloid leukaemia: 24-month minimum follow-up of the phase 3 randomised ENESTnd trial. Lancet Oncol. 2011;12(9):841-851.

47. Cortes JE, Maru A, Antonio de Souza C, et al. Bosutinib versus imatinib in newly diagnosed chronic phase chronic myeloid leukemia-BELA trial: 24-month follow-up. 53rd American Society of Hematology Annual Meeting and Exposition; December 10-13, 2011; San Diego, CA.

48. Baccarani M, Rosti G, Castagnetti F, et al. Comparison of imatinib $400 \mathrm{mg}$ and $800 \mathrm{mg}$ daily in the front-line treatment of high-risk, Philadelphia-positive chronic myeloid leukemia: a European LeukemiaNet Study. Blood. 2009;113(19):4497-4504.

49. Lipton JH, Trask PC, Cella D, et al. Health-related quality of life in newly diagnosed chronic phase chronic myelogenous leukemia patients treated with bosutinib or imatinib. American Society of Clinical Oncology Annual Meeting; June 3-7, 2011; Chicago, IL.

50. Kantarjian HM, Giles FJ, Bhalla KN, et al. Nilotinib is effective in patients with chronic myeloid leukemia in chronic phase after imatinib resistance or intolerance: 24-month follow-up results. Blood. 2011;117(4):1141-1145.

51. Cortes JE, Kantarjian HM, BrummendorfTH, et al. Safety and efficacy of bosutinib (SKI-606) in chronic phase Philadelphia chromosomepositive CML patients with resistance or intolerance to imatinib. Blood. 2011;118(7):4567-4576.

52. Saglio G, Hochhaus A, Goh YT, et al. Dasatinib in imatinib-resistant or imatinib-intolerant chronic myeloid leukemia in blast phase after 2 years of follow-up in a phase 3 study: efficacy and tolerability of 140 milligrams once daily and 70 milligrams twice daily. Cancer. 2010;116(16):3852-3861

53. Shah NP, Kim DW, Kantarjian H, et al. Potent, transient inhibition of BCR-ABL with dasatinib $100 \mathrm{mg}$ daily achieves rapid and durable cytogenetic responses and high transformation-free survival rates in chronic phase chronic myeloid leukemia patients with resistance, suboptimal response or intolerance to imatinib. Haematologica. 2010;95(2):232-240.

54. Shah N, Kim DW, Kantarjian H, et al. Dasatinib dose-optimization in chronic phase chronic myeloid leukemia (CML-CP): two-year data from CA180-034 show equivalent long-term efficacy and improved safety with $100 \mathrm{mg}$ once daily dose. Abstract 3225. 52nd American Society of Hematology Annual Meeting; December 4-7, 2010; Orlando, FL.

55. Shah N, Cortes J, Schiffer CA, et al. Four-year follow-up of patients with chronic-phase chronic myeloid leukemia (CPCML) receiving $100 \mathrm{mg}$ of dasatinib once daily. Abstract 6512 . American Society of Clinical Oncology Annual Meeting; June 4-8, 2010; Chicago, IL.

56. Shah N, Cortes J, Schiffer C, et al. Five-year follow-up of patients with imatinib-resistant or -intolerant chronic-phase chronic myeloid leukemia (CML-CP) receiving dasatinib. American Society of Clinical Oncology Annual Meeting; June 3-7, 2011; Chicago, IL.

57. Khoury HJ, Cortes JE, Kantarjian HM, et al. Bosutinib is active in chronic phase chronic myeloid leukemia after imatinib and dasatinib and/or nilotinib therapy failure. Blood. 2012;119(15):3403-3412.

58. Jabbour E, Kantarjian H, O'Brien S, et al. The achievement of an early complete cytogenetic response is a major determinant for outcome in patients with early chronic phase chronic myeloid leukemia treated with tyrosine kinase inhibitors. Blood. 2011;118(17):4541-4546. 
59. Mahon FX, Rea D, Guilhot J, et al. Discontinuation of imatinib in patients with chronic myeloid leukaemia who have maintained complete molecular remission for at least 2 years: the prospective, multicentre Stop Imatinib (STIM) trial. Lancet Oncol. 2010;11(11):1029-1035.

60. Signorovitch JE, Wu EQ, Betts KA, et al. Comparative efficacy of nilotinib and dasatinib in newly diagnosed chronic myeloid leukemia: a matching-adjusted indirect comparison of randomized trials. Curr Med Res Opin. 2011;27(6):1263-1271.
61. Cortes JE, Kim DW, Pinilla-Ibarz J, et al. Initial findings from the PACE trial: a pivotal phase 2 study of ponatinib in patients with CML and $\mathrm{Ph}+\mathrm{ALL}$ resistant or intolerant to dasatinib or nilotinib, or with the T315I mutation. Presented at 53rd American Society Hematology Annual Meeting; December 10-13, 2011; Atlanta, GA.

Journal of Blood Medicine

\section{Publish your work in this journal}

The Journal of Blood Medicine is an international, peer-reviewed, open access, online journal publishing laboratory, experimental and clinical aspects of all topics pertaining to blood based medicine including but not limited to: Transfusion Medicine; Blood collection, Donor issues, Transmittable diseases, and Blood banking logistics; Immunohematology; Artificial and alternative

\section{Dovepress}

blood based therapeutics; Hematology; Biotechnology/nanotechnology of blood related medicine; Legal aspects of blood medicine; Historical perspectives. The manuscript management system is completely online and includes a very quick and fair peer-review system. Visit http://www.dovepress.com/ testimonials.php to read real quotes from published authors.

Submit your manuscript here: http://www.dovepress.com/Journal-of-blood-medicine-journal 